\title{
Efficient Three-Party Computation from Cut-and-Choose ${ }^{\star}$
}

\author{
Seung Geol Choi ${ }^{\dagger} \quad$ Jonathan Katz ${ }^{\ddagger} \quad$ Alex J. Malozemoff ${ }^{\ddagger} \quad$ Vassilis Zikas $^{\S}$
}

\begin{abstract}
With relatively few exceptions, the literature on efficient (practical) secure computation has focused on secure two-party computation (2PC). It is, in general, unclear whether the techniques used to construct practical $2 \mathrm{PC}$ protocols - in particular, the cut-and-choose approach-can be adapted to the multi-party setting.

In this work we explore the possibility of using cut-and-choose for practical secure three-party computation. The three-party case has been studied in prior work in the semi-honest setting, and is motivated by the observation that real-world deployments of multi-party computation are likely to involve few parties. We propose a constant-round protocol for three-party computation tolerating any number of malicious parties, whose computational cost is essentially only a small constant worse than that of state-of-the-art two-party protocols.
\end{abstract}

\section{Introduction}

The past few years have seen a tremendous amount of attention devoted to making secure computation truly practical (e.g., [HEKM11, KsS12, KSS13]). With only a few exceptions [DPSZ12, DKL ${ }^{+} 13$, KSS13, however, this work has tended to focus on secure two-party computation (2PC). In the semihonest setting, a series of papers [BDNP08, HKS ${ }^{+}$10, HEKM11, HEK12] showed that Yao's garbled circuit technique [Yao86] can yield very efficient protocols for the computation of Boolean circuits. In the malicious setting, Lindell and Pinkas [LP07] initiated use of the cut-and-choose technique, also based on Yao's garbled circuits, for constructing efficient, constant-round protocols. This technique was developed further in several subsequent works Wo007, LPS08, N009, PSSW09, LP11, sS11, KsS12, HKE13, Lin13, MR13, sS13, and yields the fastest known protocols for (malicious) secure two-party computation (2PC) of Boolean circuits.

2PC protocols with malicious security can also be based on the GMW protocol [GMW87] (e.g., the TinyOT protocol [NNOB12]). Although this approach yields protocols with round complexity linear in the (multiplicative) depth of the circuit, it offers the advantage that much of the computation can be pushed to an offline, pre-processing phase that is executed before the parties receive their inputs. The subsequent online computation is very fast and uses mainly information-theoretic techniques.

\footnotetext{
* (c) IACR 2014. This article is a major revision of the version published by Springer-Verlag.

${ }^{\dagger}$ Dept. of Computer Science, United States Naval Academy. Portions of this work were done while at the University of Maryland. Email: choi@usna.edu

${ }^{\ddagger}$ Dept. of Computer Science, University of Maryland. Email: \{jkatz, amaloz\}@cs.umd.edu

${ }^{\S}$ Dept. of Computer Science, ETH Zurich. Portions of this work were done while at the University of Maryland and the University of California, Los Angeles. Email: vzikas@inf.ethz.ch
} 
In the setting of multi-party computation (MPC) with security against an arbitrary number of corruptions, the situation is somewhat different. While there has been much recent work on optimizing MPC for semi-honest adversaries [BDNP08, BLW08, BCD ${ }^{+}$09, DGKN09, BSMD10, BTW12, $\mathrm{CHK}^{+} 12$, less work has focused on security against malicious corruptions. The work of Ishai, Prabhakaran, and Sahai [IPS08] gives protocols with good asymptotic efficiency; however, despite some promising optimizations [LOP11, it has not yet produced practical instantiations. The SPDZ protocol [BDOZ11, $\mathrm{DKL}^{+} 12$, DPSZ12, $\mathrm{DKL}^{+} 13$, KSS13, which handles arithmetic circuits, has extremely fast online running time at the cost of a very slow offline phase. However, unlike protocols based on garbled circuits, SPDZ runs for a linear (instead of constant) number of (online) rounds, and in each such round every party needs to utilize a broadcast channel. To our knowledge, SPDZ's implementation experiments [DKL ${ }^{+} 12$, DPSZ12, $\mathrm{DKL}^{+} 13$ ] were run on a local-area network where physical broadcast is available, and thus the delay due to accounting for round-timeouts and/or running a multi-party broadcasting protocol when operating in a wide-area network environment has not been taken into account. This delay may be non-trivial depending on circumstances: Schneider and Zohner [SZ13] have shown that as the latency between machines increases, the cost of each round becomes more and more significant.

Finally, the work of Goyal, Mohassel, and Smith GMS08 uses the (two-party) cut-and-choose technique to construct a multi-party protocol; however, security only holds in the weaker covert security setting [AL07.

Multi-Party Computation for a Small Number of Parties. Research on secure computation has traditionally been divided into two classes: work focusing on two-party computation, and work focusing on multi-party computation for an arbitrary number of parties 11 Yet, in practice, it seems that the most likely scenarios for secure MPC would involve a small number of parties. In general, as the number of parties increases, the cost of communication amongst the parties increases as well. In a wide-area network setting, this may have a huge impact on the running time of the protocol.

In addition, the three-party setting is interesting in its own right. For example, we can consider the following use-case. Suppose the government would like to run some privacy preserving computation on a company's dataset, such as flight manifests. Now, suppose the public does not trust that these parties are not colluding. Thus, we could add a third party, trusted by the public, into the computation to enforce that the two main parties are not simply sharing all their information.

Our Contributions. Motivated by these observations, we initiate the study of efficient three-party computation in the malicious model, tolerating an arbitrary number of corruptions. We construct the first practical, constant-round protocol for secure three-party computation of Boolean circuits. Our protocol uses player-simulation techniques in order to compile existing (cut-and-choose-based) 2PC protocols into three-party protocols. We instantiate our compiler with state-of-the-art $2 \mathrm{PC}$ constructions and show that the addition of a third party comes at the cost of roughly a factor eight overhead over the underlying $2 \mathrm{PC}$ protocol in terms of computation, and a factor sixteen overhead in terms of communication. This running time appears to be superior to existing state-of-the-art MPC protocols in terms of start-to-finish running time. Of course, computing the exact overhead requires implementations of both our protocol and the underlying $2 \mathrm{PC}$ protocol and is a subject of future research. As a further optimization point, our protocol makes only three calls overall to a broadcast channel (one with each party as sender), as opposed to existing practical MPC

\footnotetext{
${ }^{1}$ Here we are interested in protocols tolerating an arbitrary number of corruptions. One could further distinguish work on MPC that assumes an honest majority.
} 
solutions (for more than two parties) which use broadcast for communicating all protocol messages. This may be important in certain wide-area network settings where communication (and broadcast specifically) is very expensive. The most efficient instantiation of our protocol requires the random oracle model. As a downside, our protocol does not currently support free-XOR [KS08] or garbled row reduction PSSW09; we leave such developments as future work.

Overview of Our Protocol. Denote the three parties by $P_{1}, P_{2}$, and $P_{3}$. The high-level idea of our construction is to execute a two-party protocol $\widehat{\pi}$, where one of the two parties (say $\widehat{P}_{1}$ ) is emulated by $P_{1}$ and $P_{2}$ via a two-party protocol $\pi$, and the other party is played by $P_{3}$.

Clearly, naïvely applying the above idea yields an inefficient construction even when state-of-theart 2PC protocols are used for $\pi$ and $\widehat{\pi}$. Assume, for example, that the most efficient 2PC protocol is used for both $\pi$ and $\widehat{\pi}$, where $\pi$ simply computes the circuit of $\widehat{P}_{1}$ among $P_{1}$ and $P_{2}$. The security of the resulting construction follows trivially from the composition theorem. However, unless the size of the circuit is very small, this approach results in a huge blowup on the overall runtime; in particular, if $t$ is the time $\pi$ needs to compute the circuit of $\widehat{P}_{1}$ and $\widehat{t}$ is the time that $\widehat{\pi}$ needs to compute the three-party circuit, then the runtime of the above naïve construction is $t \cdot \hat{t}$, yielding at least a quadratic blowup.

Emulating the Sender Versus Emulating the Receiver. In most cut-and-choose-based 2PC protocols, the parties have distinct roles: one is the sender, or circuit generator, and the other is the receiver, or circuit verifier. One might be tempted to think that, because the role of the verifier in the protocol is more "passive" (in the sense that the computation is less complicated), the most natural approach would be to emulate the verifier among $P_{1}$ and $P_{2}$ (and have $P_{3}$ locally do the heavier work doing circuit generation and opening over broadcast). This seemingly direct approach fails as one needs a mechanism for $P_{1}$ and $P_{2}$ to include their inputs into the garbled circuits. Clearly, doing so by having $P_{1}$ first receive his input-keys via OT (as in the original Yao-based constructions) and then handing them to $P_{2}$ yields an insecure protocol; indeed, an adversary corrupting $P_{2}$ and $P_{3}$ can then trivially learn $P_{1}$ 's inputs.

Instead, in this work we have $P_{1}$ and $P_{2}$ emulate the sender, and we have $P_{3}$ play the role of the receiver. More precisely, we adapt the distributed circuit-garbling technique [BMR90, DI05] to the two-party setting, allowing $P_{1}$ and $P_{2}$ to compute a sharing of a garbled circuit which they then reconstruct for $P_{3}$. By appropriate optimizations, we ensure that distributed garbling requires $P_{1}$ and $P_{2}$ to compute and communicate roughly as much as the sender in an execution of the Yao protocol (plus some OT calls per gate); $P_{3}$ needs to do nothing during the circuit garbling. Most interestingly, our construction features a mechanism which allows $P_{3}$ to receive the keys corresponding to his input bits for evaluating the garbled circuit by only one invocation of OT per input-bit with each of $P_{1}$ and $P_{2}$.

Our distributed garbling scheme is secure against malicious adversaries, which ensures that an adversary corrupting only one of the parties $P_{1}$ or $P_{2}$ cannot produce a maliciously constructed garbled circuit. In order to protect against an adversary who corrupts both $P_{1}$ and $P_{2}$, we rely on the cut-and-choose technique. We give concrete instantiations (in the random oracle model) of our protocol using a combination of two 2PC protocols by Lindell and Pinkas [LP07, LP11], as well as a construction based on the more recent protocol by Lindell [Lin13] which drastically reduces the number of circuit garblings required for cut-and-choose.

Interestingly, the cut-and-choose technique does not only protect against corrupting both $P_{1}$ and $P_{2}$, but allows a considerable efficiency improvement. More precisely, it allows us to avoid using costly authenticated shares (towards $P_{3}$ ) for the computed (shared) garbled circuit. Instead, 
our distributed garbling scheme outputs, even in the malicious setting, a plain two-out-of-two sum sharing of the garbled circuit.

The Security Model. We assume the reader is familiar with the simulation paradigm GMW87, Can00, Can01, Gol09. For simplicity we restrict ourselves to computation of non-reactive functions, also known as secure function evaluation. We consider a static adversary that gets to choose the corrupted parties at the beginning of the protocol. Without loss of generality, we assume that the circuit to be computed is deterministic and has a single output received by one of the parties. We point out that although we use the language of Canetti [Can00, all our proofs use straight-line black-box simulators, which makes our protocols secure in the Universal Composition framework Can01].

Outline. In Section 2 we cover preliminary topics. In Section 3 we describe our two-party distributed garbling scheme, and in Section 4 we discuss our three party protocol. In Section 5 we show how to instantiate the various two-party functionalities we utilize in our protocols.

\section{Preliminaries}

We let $k$ denote the computational security parameter and let $s$ denote the statistical security parameter. We use $x \leftarrow \$ S$ to denote choosing a value $x$ uniformly at random from the set $S$, and use $\|$ to denote concatenation.

Circuit Notation. We follow the circuit notation of Bellare et al. [BHR12]. Let ( $\mathrm{n}, \mathrm{m}, \mathrm{q}, L, R, G) \leftarrow$ $C$ be a circuit, where $\mathrm{n}$ is the number of input wires, $\mathrm{m}$ is the number of output wires, and $\mathrm{q}$ is the number of gates, where each gate is indexed by its output wire. Thus, the total number of wires in the circuit is $\mathrm{n}+\mathrm{q}$. The numbering of wires starts with the inputs and ends with the outputs; i.e., we have inputs $\{1, \ldots, \mathrm{n}\}$ and outputs $\{\mathrm{n}+\mathrm{q}-\mathrm{m}+1, \ldots, \mathrm{n}+\mathrm{q}\}$. The function $L$ (resp., $R$ ) takes as input a gate index and returns the left (resp., right) input wire to the gate. We require $L(\gamma)<R(\gamma)<\gamma$ for any gate index $\gamma$. The function $G$ encodes the functionality of a given gate, e.g., $G_{\gamma}(0,1)=0$ if the gate with index $\gamma$ is an AND gate. Because we consider circuits with inputs from multiple parties, let $\left\{\mathrm{n}_{i-1}+1, \ldots, \mathrm{n}_{i}\right\}$ denote the input wires "controlled" by party $P_{i}$, with $\mathrm{n}_{0}=0$.

We denote input gates as those gates with one or more input wires, inner gates as those gates with no input or output wires, and output gates as those gates with an output wire.

Secret Sharing. Our constructions use two-out-of-two secret sharing. In the semi-honest setting, we use a standard (linear) sharing of strings: the secret $x \in\{0,1\}^{*}$ is split into two random summands $x_{1}$ and $x_{2}$ such that $x_{1} \oplus x_{2}=x$, with $P_{i}$ holding the summand $x_{i}$. We denote the sharing of $x$ by $[x]=\left([x]^{(1)},[x]^{(2)}\right)$, where we refer to each $[x]^{(i)}=x_{i}$ as $P_{i}$ 's share of $x$. This sharing is linear: If $[x]$ and $[y]$ are sharings of $x$ and $y$ respectively, then $[x] \oplus[y]$ is a sharing of $x \oplus y$; that is, $[x \oplus y]=[x] \oplus[y]$ and thus $P_{i}$ can locally compute his share as $[x \oplus y]^{(i)}=[x]^{(i)} \oplus[y]^{(i)}$. It is straight-forward to verify that the above secret-sharing is private provided that the summands $x_{1}$ and $x_{2}$ are uniformly chosen (restricted only on $x_{1} \oplus x_{2}=x$ ); i.e., any single share $[x]^{(i)}$ contains no information about the secret $x$. Reconstructing a sharing $[x]$ is easily done by having each party announce his share $[x]^{(i)}$ and taking $x$ to be the exclusive-or of the announced shares.

Our protocols use shares of two types of secrets: $k$-bit strings $x \in\{0,1\}^{k}$ and bits $b \in\{0,1\}$. For clarity in the presentation, we use the bracket notation introduced above for sharings of $x \in\{0,1\}^{k}$, 
and use the notation $\langle\cdot\rangle$ for sharings of bits; i.e., if $b \in\{0,1\}$ then a sharing of $b$ is denoted as $\langle b\rangle=\left(\langle b\rangle^{(1)},\langle b\rangle^{(2)}\right)$.

In the malicious setting we need the sharings of bits to be authenticated; i.e., in addition to his summand $b_{i}$, each party $P_{i}$ holds an authentication tag $t_{i}$ for a Message Authentication Code (MAC), with another party $P_{j}$ holding the corresponding verification key $k_{j}$. More precisely, in a sharing $\langle b\rangle=\left(\langle b\rangle^{(1)},\langle b\rangle^{(2)}\right)$ of $b$, each party's share is now a tuple $\langle b\rangle^{(i)}:=\left(b_{i}, t_{i}, k_{j}\right)$, where $b_{1} \oplus b_{2}=b$, and $t_{i}$ is a valid MAC on $b_{i}$ with key $k_{j}$. This ensures that the adversary cannot make the reconstruction output any value other than the secret $b$. In particular, to reconstruct some sharing $\langle b\rangle=\left(\langle b\rangle^{(1)},\langle b\rangle^{(2)}\right)$, each party $P_{i}$ first announces his summand $b_{i}$ and the corresponding authentication tag $t_{i}$; subsequently, each party $P_{i}$ checks that the other party $P_{j}$ announced a validly authenticated summand matching his own verification key and if this is not the case he rejects. The inability of an adversarial $P_{i}$ to announce a summand other than $b_{i}$ follows from the unforgeability of the MAC, as $P_{i}$ does not know the key $k_{j}$ matching his authentication tag.

We also assume this authentication is linear in the following sense: Given $\langle b\rangle$ and $\left\langle b^{\prime}\right\rangle$, the parties can compute $\langle b\rangle \oplus\left\langle b^{\prime}\right\rangle$ locally. Namely, $\langle b\rangle \oplus\left\langle b^{\prime}\right\rangle=\left(\left\langle b \oplus b^{\prime}\right\rangle^{(1)},\left\langle b \oplus b^{\prime}\right\rangle^{(2)}\right)$, where $\left\langle b \oplus b^{\prime}\right\rangle^{(i)}=$ $\left(b_{i} \oplus b_{i}^{\prime}, t_{i} \oplus t_{i}^{\prime}, k_{j} \oplus k_{j}^{\prime}\right)$ is a valid authentication. We show how to construct such an authenticated sharing in Section 5 .

\section{Two-Party Distributed Garbling Scheme}

In this section we describe our construction of a two-party distributed garbling scheme. Our protocol combines the standard Yao garbling circuit technique with the distributed garbling ideas from Damgård and Ishai [DI05]. The main idea is the following: The players jointly compute a garbled circuit, where the gates are garbled by use of a distributed encryption scheme which takes, for each encryption, one key from each party.

In more detail, our construction is described in several steps. As the first step, in Section 3.1 we give a description of our garbling scheme; i.e., the code of the sender in our version of Yao's protocol. This section gives the reader familiarity with our notation and is used as a reference in the distributed protocol. Next, in Section 3.2 we describe an efficient (semi-honest) protocol that allows parties $P_{1}$ and $P_{2}$ to securely emulate the circuit-garbling procedure from Section 3.1. Finally, in Section 3.3 , we show how to make the garbling procedure maliciously secure.

\subsection{Single-Party Garbling Scheme}

Our garbling scheme is a slight variant of the protocol by Damgård and Ishai [DI05] adapted to two parties. This should be regarded as an initial step towards our ultimate goal of a distributed garbling scheme. Here, we describe the high-level construction; see Figure 1 for the detailed protocol.

We associate two random keys $K_{w, 0}, K_{w, 1}$ with each wire $w$ in the circuit; key $K_{w, 0}$ corresponds to the value ' 0 ' and $K_{w, 1}$ corresponds to the value ' 1 '. Each key $K_{w, b}$ consists of two sub-keys $s_{w, b}^{1}$ and $s_{w, b}^{2}$; that is, $K_{w, b}=\left(s_{w, b}^{1}, s_{w, b}^{2}\right)$. In addition, for each wire $w$ we choose a random mask bit $\lambda_{w}$. Each key has an associated tag, derived from the mask bit, which acts as a blinding of the true value the key represents.

Now, consider gate $G_{\gamma}$ in the circuit with input wires $\alpha$ and $\beta$. The garbled gate of $G_{\gamma}$ consists of an array of four encryptions: for each $\left(b_{\alpha}, b_{\beta}\right) \in\{0,1\} \times\{0,1\}$, the row $\left(b_{\alpha}, b_{\beta}\right)$ consists of an encryption of $K_{\gamma, G_{\gamma}\left(b_{\alpha} \oplus \lambda_{\alpha}, b_{\beta} \oplus \lambda_{\beta}\right) \oplus \lambda_{\gamma}}$ and its corresponding tag $G_{\gamma}\left(b_{\alpha} \oplus \lambda_{\alpha}, b_{\beta} \oplus \lambda_{\beta}\right) \oplus \lambda_{\gamma}$ under keys 
Auxiliary Inputs: Security parameter $k$, circuit $(\mathrm{n}, \mathrm{m}, \mathrm{q}, L, R, G) \leftarrow C$.

1. Generate masks:

- Generate input and inner mask bits: For $w \in\{1, \ldots, \mathbf{n}+\mathbf{q}-\mathbf{m}\}$ : generate $\lambda_{w} \leftarrow \$\{0,1\}$.

- Generate output mask bits: For $w \in\{\mathbf{n}+\mathbf{q}-\mathbf{m}+1, \ldots, \mathbf{n}+\mathbf{q}\}$ : generate $\lambda_{w} \leftarrow 0$.

2. Generate sub-keys:

- For wires $w \in\{1, \ldots, \mathbf{n}+\mathbf{q}\}$ and $b \in\{0,1\}$ : generate sub-keys $s_{w, b}^{1}, s_{w, b}^{2} \leftarrow \$\{0,1\}^{k}$.

3. Construct garbled circuit:

- Construct garbled gates: For gates $\gamma \in\{\mathrm{n}+1, \ldots, \mathrm{n}+\mathrm{q}\}$, do the following: Let $\alpha \leftarrow L(\gamma)$ and $\beta \leftarrow R(\gamma)$ be the index of the left and right input wires, respectively, of the gate indexed by $\gamma$. Letting $K_{w, b}=\left(s_{w, b}^{1}, s_{w, b}^{2}\right)$, compute the following:

$$
\begin{aligned}
& P[\gamma, 0,0] \leftarrow \operatorname{Enc}_{K_{\alpha, 0}, K_{\beta, 0}}\left(K_{\gamma, G_{\gamma}\left(\lambda_{\alpha}, \lambda_{\beta}\right) \oplus \lambda_{\gamma}} \| G_{\gamma}\left(\lambda_{\alpha}, \lambda_{\beta}\right) \oplus \lambda_{\gamma}\right) \\
& P[\gamma, 0,1] \leftarrow \operatorname{Enc}_{K_{\alpha, 0}, K_{\beta, 1}}\left(K_{\gamma, G_{\gamma}\left(\lambda_{\alpha}, \lambda_{\beta} \oplus 1\right) \oplus \lambda_{\gamma}} \| G_{\gamma}\left(\lambda_{\alpha}, \lambda_{\beta} \oplus 1\right) \oplus \lambda_{\gamma}\right) \\
& P[\gamma, 1,0] \leftarrow \operatorname{Enc}_{K_{\alpha, 1}, K_{\beta, 0}}\left(K_{\gamma, G_{\gamma}\left(\lambda_{\alpha} \oplus 1, \lambda_{\beta}\right) \oplus \lambda_{\gamma}} \| G_{\gamma}\left(\lambda_{\alpha} \oplus 1, \lambda_{\beta}\right) \oplus \lambda_{\gamma}\right) \\
& P[\gamma, 1,1] \leftarrow \operatorname{Enc}_{K_{\alpha, 1}, K_{\beta, 1}}\left(K_{\gamma, G_{\gamma}\left(\lambda_{\alpha} \oplus 1, \lambda_{\beta} \oplus 1\right) \oplus \lambda_{\gamma}} \| G_{\gamma}\left(\lambda_{\alpha} \oplus 1, \lambda_{\beta} \oplus 1\right) \oplus \lambda_{\gamma}\right)
\end{aligned}
$$

4. Output circuit:

- $\quad$ Set $G C \leftarrow(\mathrm{n}, \mathrm{m}, \mathrm{q}, L, R, P)$, and output:

$$
\left(G C,\left\{\left(s_{w, b \oplus \lambda_{w}}^{1}, s_{w, b \oplus \lambda_{w}}^{2}, b \oplus \lambda_{w}\right): w \in\{1, \ldots, \mathbf{n}\}, b \in\{0,1\}\right\}\right) .
$$

Figure 1: Circuit garbling scheme.

$K_{\alpha, b_{\alpha}}$ and $K_{\beta, b_{\beta}}$. Let $P$ denote a table that stores all the garbled gates; in particular, the entry $P\left[\gamma, b_{\alpha}, b_{\beta}\right]$ contains an encryption corresponding to row $\left(b_{\alpha}, b_{\beta}\right)$ of the garbled gate for $G_{\gamma}$.

Evaluation proceeds as follows. Let $\alpha$ and $\beta$ be input wires connected to gate $G$ with index $\gamma$.

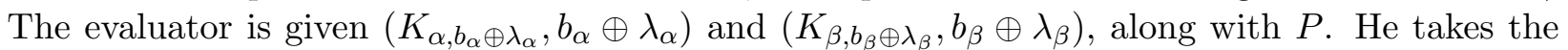
row $P\left[\gamma, b_{\alpha} \oplus \lambda_{\alpha}, b_{\beta} \oplus \lambda_{\beta}\right]$ and decrypts it using the keys $K_{\alpha, b_{\alpha} \oplus \lambda_{\alpha}}$ and $K_{\beta, b_{\beta} \oplus \lambda_{\beta}}$, resulting in $\left(K_{\gamma, G\left(b_{\alpha}, b_{\beta}\right) \oplus \lambda_{\gamma}}, G\left(b_{\alpha}, b_{\beta}\right) \oplus \lambda_{\gamma}\right)$. It is straightforward to verify that by continuing this evaluation, the output of each gate will be revealed masked by its corresponding mask. By picking masks of the output wires to be ' 0 ' we ensure that the evaluator receives the (unmasked) output of the circuit.

\subsection{Distributing the Garbling Scheme Between Two Parties}

We now show how to emulate the above garbling scheme between two parties in the semi-honest setting. We assume the parties have access to the following two-party ideal functionalities:

- Gate computation $\mathcal{F}_{\text {gate }}^{G}(\langle a\rangle,\langle b\rangle)$ : The functionality takes as input sharings $\langle a\rangle$ and $\langle b\rangle$ of bits $a$ and $b$, respectively, and is parameterized by a binary gate $G$; it outputs a sharing $\langle G(a, b)\rangle$ of the output of $G$ on input $(a, b)$.

- One-out-of-two oblivious secret sharing $\mathcal{F}_{\text {oshare }}^{i}\left(\langle b\rangle, m_{0}, m_{1}\right)$ : The functionality takes as input a sharing $\langle b\rangle$ of a bit $b$ (i.e., each party inputs his share), along with two messages $m_{0}, m_{1}$ from $P_{i}$, and outputs a random two-out-of-two sharing $\left[m_{b}\right]$ of $m_{b}$.

- Constant bit sharing $\mathcal{F}_{\text {const }}^{b}()$ : The functionality is parameterized by a bit $b \in\{0,1\}$, and outputs a random sharing $\langle b\rangle$ of $b$. 
- Random bit sharing $\mathcal{F}_{\text {rand }}()$ : The functionality chooses a random bit $r \leftarrow \$\{0,1\}$ and computes and outputs a random sharing $\langle r\rangle$ of $r$.

- Bit secret sharing $\mathcal{F}_{\mathbf{s} \mathbf{s}}^{i}(b)$ : The functionality takes input bit $b \in\{0,1\}$ from $P_{i}$ and outputs a random two-out-of-two sharing $\langle b\rangle$ of $b$.

Each of these can be instantiated efficiently in the semi-honest setting; see Appendix A and Appendix B for details.

Distributed Encryption Scheme. We utilize the distributed encryption scheme by Damgård and Ishai DI05]. Suppose the message and the key for the encryption scheme are distributed as follows:

- The message $m$ is secret-shared; i.e., $P_{1}$ and $P_{2}$ hold $[m]^{(1)}$ and $[m]^{(2)}$, respectively.

- The encryption key $K=\left(s^{1}, s^{2}\right)$ is distributed such that $P_{1}$ and $P_{2}$ hold $s^{1}$ and $s^{2}$, respectively.

The encryption of the secret-shared message $m$ with tweak $T$ under key $K=\left(s^{1}, s^{2}\right)$ is:

$$
\operatorname{Enc}_{K}^{T}([m])=\left(\operatorname{Enc}_{s^{1}, T}^{1}\left([m]^{(1)}\right), \operatorname{Enc}_{s^{2}, T}^{2}\left([m]^{(2)}\right)\right)=\left([m]^{(1)} \oplus F_{s^{1}}^{1}(T),[m]^{(2)} \oplus F_{s^{2}}^{1}(T)\right)
$$

where $F_{k}^{1}$ is a PRF keyed by key $k$. To decrypt a ciphertext $c:=\operatorname{Enc}_{K}^{T}(m)$, each party $P_{i}$ sends his sub-key $s^{i}$ to the decrypter, who uses them to recover the shares of $m$ and reconstruct $m$.

Double encryption is defined analogously. For keys $K_{\alpha}=\left(s_{\alpha}^{1}, s_{\alpha}^{2}\right)$ and $K_{\beta}=\left(s_{\beta}^{1}, s_{\beta}^{2}\right)$, where $P_{i}$ holds $\left(s_{\alpha}^{i}, s_{\beta}^{i}\right)$, encryption with tweak $T$ works as follows:

$$
\operatorname{Enc}_{K_{\alpha}, K_{\beta}}^{T}([m])=\left([m]^{(1)} \oplus F_{s_{\alpha}^{1}}^{1}(T) \oplus F_{s_{\beta}^{1}}^{2}(T),[m]^{(2)} \oplus F_{s_{\alpha}^{2}}^{1}(T) \oplus F_{s_{\beta}^{2}}^{2}(T)\right) .
$$

Distributed Garbling Scheme. We now give a high-level description of our two-party distributed garbling scheme $\Pi_{\mathbf{G C}}\left(P_{1}, P_{2}\right)$; see Figure 2 for the detailed description. As before, for each wire $w$ in the circuit we associate keys $K_{w, 0}=\left(s_{w, 0}^{1}, s_{w, 0}^{2}\right)$ and $K_{w, 1}=\left(s_{w, 1}^{1}, s_{w, 1}^{2}\right)$ corresponding to bits ' 0 ' and ' 1 ', respectively. However, in the distributed setting, each sub-key is only known to one of the two parties; i.e., $P_{i}$ only knows $\left(s_{w, 0}^{i}, s_{w, 1}^{i}\right)$. Each wire is also associated with a mask bit $\lambda_{w}$ which is secret shared between the two parties such that no party knows $\lambda_{w}$.

Consider gate $G_{\gamma}$ in the circuit with input wires indexed by $\alpha$ and $\beta$. As in the non-distributed case, we construct an array containing four rows corresponding to a random permutation of the four possible outcomes of gate $G_{\gamma}$ applied to bits $b_{\alpha}$ and $b_{\beta}$. However, in the distributed case neither party should know what is being encrypted. Recall that in the non-distributed setting, the circuit generator can easily compute $G_{\gamma}\left(\lambda_{\alpha} \oplus b_{\alpha}, \lambda_{\beta} \oplus b_{\beta}\right)$ to construct the array. However, in the distributed setting, neither party knows (and should not know) $\lambda_{\alpha}$ or $\lambda_{\beta}$. Thus, the parties utilize the $\mathcal{F}_{\text {gate }}$ functionality, which takes as input the shares $\left\langle\lambda_{\alpha}\right\rangle \oplus\left\langle b_{\alpha}\right\rangle$ and $\left\langle\lambda_{\beta}\right\rangle \oplus\left\langle b_{\beta}\right\rangle$, and computes a sharing of $G_{\gamma}\left(\lambda_{\alpha} \oplus b_{\alpha}, \lambda_{\beta} \oplus b_{\beta}\right)$. Let $\left\langle\sigma_{\gamma, b_{\alpha}, b_{\beta}}\right\rangle=\mathcal{F}_{\text {gate }}^{G}\left(\left\langle b_{\alpha}\right\rangle \oplus\left\langle\lambda_{\alpha}\right\rangle,\left\langle b_{\beta}\right\rangle \oplus\left\langle\lambda_{\beta}\right\rangle\right) \oplus\left\langle\lambda_{\gamma}\right\rangle$. The value

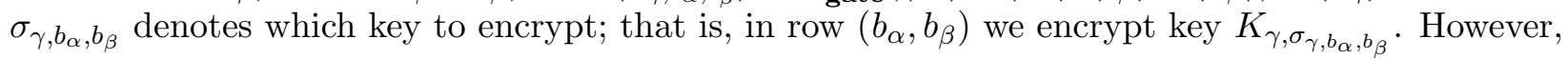
we must still enforce that neither party knows what key $K_{\gamma, \sigma_{\gamma, b_{\alpha}, b_{\beta}}}$ represents. We handle this by utilizing another functionality, $\mathcal{F}_{\text {oshare }}$. For each of the four $\sigma_{\gamma, b_{\alpha}, b_{\beta}}$ values, and for each party $P_{i}$, the parties compute $\mathcal{F}_{\text {oshare }}^{i}\left(\left\langle\sigma_{\gamma, b_{\alpha}, b_{\beta}}\right\rangle, s_{\gamma, 0}^{i}, s_{\gamma, 1}^{i}\right)$. This produces a share of the appropriate sub-key for party $P_{i}$, with the crucial fact that $P_{i}$ does not know which of his sub-keys was shared. The results of $\mathcal{F}_{\text {oshare }}$ are used as the shares to be encrypted. 


\section{Protocol $\Pi_{\mathbf{G C}}\left(P_{1}, P_{2}\right)$}

Auxiliary Inputs: Security parameter $k$, circuit $(\mathrm{n}, \mathrm{m}, \mathrm{q}, L, R, G) \leftarrow C$.

Parties $P_{1}$ and $P_{2}$ generate $\langle 1\rangle \leftarrow \mathcal{F}_{\text {const }}^{1}$, which they use throughout the protocol.

1. Generate mask bits:

- Generate masks for $P_{1}$ 's inputs: For $w \in\left\{1, \ldots, \mathbf{n}_{1}\right\}: P_{1}$ generates $\lambda_{w} \leftarrow \$\{0,1\}$ and computes $\left\langle\lambda_{w}\right\rangle \leftarrow \mathcal{F}_{\mathbf{s s}}^{1}\left(\lambda_{w}\right)$.

- Generate masks for $P_{2}$ 's inputs: For $w \in\left\{\mathrm{n}_{1}+1, \ldots, \mathbf{n}\right\}: P_{2}$ generates $\lambda_{w} \leftarrow \$\{0,1\}$ and computes $\left\langle\lambda_{w}\right\rangle \leftarrow \mathcal{F}_{\mathbf{s s}}^{2}\left(\lambda_{w}\right)$.

- Generate masks for inner wires: For $w \in\{\mathrm{n}+1, \ldots, \mathrm{n}+\mathrm{q}-\mathrm{m}\}$ : generate $\left\langle\lambda_{w}\right\rangle \leftarrow \mathcal{F}_{\text {rand }}$.

- Generate masks for output wires: For $w \in\{\mathrm{n}+\mathrm{q}-\mathrm{m}+1, \ldots, \mathrm{n}+\mathrm{q}\}$ : generate $\left\langle\lambda_{w}\right\rangle \leftarrow \mathcal{F}_{\text {const }}^{0}{ }^{a}$

2. Generate sub-keys:

- For $w \in\{1, \ldots, \mathrm{n}+\mathrm{q}\}$ and $b \in\{0,1\}: P_{i}$ generates sub-keys $s_{w, b}^{i} \leftarrow \$\{0,1\}^{k}$.

3. Construct garbled circuit:

- For $\gamma \in\{\mathrm{n}+1, \ldots, \mathrm{n}+\mathrm{q}\}$ :

Let $\alpha \leftarrow L(\gamma)$ and $\beta \leftarrow R(\gamma)$ be the indices of the left and right input wires, respectively, of the gate indexed by $\gamma$. Compute the following selector bits:

$$
\begin{array}{lll}
\left\langle\sigma_{\gamma, 0,0}\right\rangle \leftarrow \mathcal{F}_{\text {gate }}^{G_{\gamma}}\left(\left\langle\lambda_{\alpha}\right\rangle,\left\langle\lambda_{\beta}\right\rangle\right) \oplus\left\langle\lambda_{\gamma}\right\rangle & \left\langle\sigma_{\gamma, 0,1}\right\rangle \leftarrow \mathcal{F}_{\text {gate }}^{G_{\gamma}}\left(\left\langle\lambda_{\alpha}\right\rangle,\left\langle\lambda_{\beta}\right\rangle \oplus\langle 1\rangle\right) \oplus\left\langle\lambda_{\gamma}\right\rangle \\
\left\langle\sigma_{\gamma, 1,0}\right\rangle \leftarrow \mathcal{F}_{\text {gate }}^{G_{\gamma}}\left(\left\langle\lambda_{\alpha}\right\rangle \oplus\langle 1\rangle,\left\langle\lambda_{\beta}\right\rangle\right) \oplus\left\langle\lambda_{\gamma}\right\rangle & \left\langle\sigma_{\gamma, 1,1}\right\rangle \leftarrow \mathcal{F}_{\text {gate }}^{G_{\gamma}}\left(\left\langle\lambda_{\alpha}\right\rangle \oplus\langle 1\rangle,\left\langle\lambda_{\beta}\right\rangle \oplus\langle 1\rangle\right) \oplus\left\langle\lambda_{\gamma}\right\rangle .
\end{array}
$$

Next, compute sharings of the appropriate sub-keys to use for each row:

$$
\begin{array}{rlrl}
{\left[\hat{s}_{\gamma, 0,0}^{1}\right]} & \leftarrow \mathcal{F}_{\text {oshare }}^{1}\left(\left\langle\sigma_{\gamma, 0,0}\right\rangle, s_{\gamma, 0}^{1}, s_{\gamma, 1}^{1}\right), & {\left[\hat{s}_{\gamma, 0,0}^{2}\right] \leftarrow \mathcal{F}_{\text {oshare }}^{2}\left(\left\langle\sigma_{\gamma, 0,0}\right\rangle, s_{\gamma, 0}^{2}, s_{\gamma, 1}^{2}\right)} \\
\left.\hat{s}_{\gamma, 0,1}^{1}\right] \leftarrow \mathcal{F}_{\text {oshare }}^{1}\left(\left\langle\sigma_{\gamma, 0,1}\right\rangle, s_{\gamma, 0}^{1}, s_{\gamma, 1}^{1}\right), & {\left[\hat{s}_{\gamma, 0,1}^{2}\right] \leftarrow \mathcal{F}_{\text {oshare }}^{2}\left(\left\langle\sigma_{\gamma, 0,1}\right\rangle, s_{\gamma, 0}^{2}, s_{\gamma, 1}^{2}\right)} \\
\left.\hat{s}_{\gamma, 1,0}^{1}\right] \leftarrow \mathcal{F}_{\text {oshare }}^{1}\left(\left\langle\sigma_{\gamma, 1,0}\right\rangle, s_{\gamma, 0}^{1}, s_{\gamma, 1}^{1}\right), & \left.\hat{s}_{\gamma, 1,0}^{2}\right] \leftarrow \mathcal{F}_{\text {oshare }}^{2}\left(\left\langle\sigma_{\gamma, 1,0}\right\rangle, s_{\gamma, 0}^{2}, s_{\gamma, 1}^{2}\right) \\
\left.\hat{s}_{\gamma, 1,1}^{1}\right] \leftarrow \mathcal{F}_{\text {oshare }}^{1}\left(\left\langle\sigma_{\gamma, 1,1}\right\rangle, s_{\gamma, 0}^{1}, s_{\gamma, 1}^{1}\right), & {\left[\hat{s}_{\gamma, 1,1}^{2}\right] \leftarrow \mathcal{F}_{\text {oshare }}^{2}\left(\left\langle\sigma_{\gamma, 1,1}\right\rangle, s_{\gamma, 0}^{2}, s_{\gamma, 1}^{2}\right) .}
\end{array}
$$

Finally, compute the distributed encryptions of the (permuted) sub-keys and selector bits. That is, letting $K_{w, b}=\left(s_{w, b}^{1}, s_{w, b}^{2}\right)$, compute:

$$
\begin{aligned}
& P[\gamma, 0,0]=\left(P^{1}[\gamma, 0,0], P^{2}[\gamma, 0,0]\right) \leftarrow \operatorname{Enc}_{K_{\alpha}, 0, K_{\beta, 0}}^{\gamma\|0\| 0}\left(\left[\hat{s}_{\gamma, 0,0}^{1}\right]\left\|\left[\hat{s}_{\gamma, 0,0}^{2}\right]\right\|\left\langle\sigma_{\gamma, 0,0}\right\rangle\right), \\
& P[\gamma, 0,1]=\left(P^{1}[\gamma, 0,1], P^{2}[\gamma, 0,1]\right) \leftarrow \operatorname{Enc}_{K_{\alpha, 0} \| 1}^{\gamma \| K_{\beta, 1}}\left(\left[\hat{s}_{\gamma, 0,1}^{1}\right]\left\|\left[\hat{s}_{\gamma, 0,1}^{2}\right]\right\|\left\langle\sigma_{\gamma, 0,1}\right\rangle\right), \\
& P[\gamma, 1,0]=\left(P^{1}[\gamma, 1,0], P^{2}[\gamma, 1,0]\right) \leftarrow \operatorname{Enc}_{K_{\alpha}, 1, K_{\beta, 0}}^{\gamma\|1\| 0}\left(\left[\hat{s}_{\gamma, 1,0}^{1}\right]\left\|\left[\hat{s}_{\gamma, 1,0}^{2}\right]\right\|\left\langle\sigma_{\gamma, 1,0}\right\rangle\right), \\
& P[\gamma, 1,1]=\left(P^{1}[\gamma, 1,1], P^{2}[\gamma, 1,1]\right) \leftarrow \operatorname{Enc}_{K_{\alpha, 1}, K_{\beta, 1}}^{\gamma\|1\| 1}\left(\left[\hat{s}_{\gamma, 1,1}^{1}\right]\left\|\left[\hat{s}_{\gamma, 1,1}^{2}\right]\right\|\left\langle\sigma_{\gamma, 1,1}\right\rangle\right) .
\end{aligned}
$$

\section{Output circuit:}

- Let $G C^{i} \leftarrow\left(\mathrm{n}, \mathrm{m}, \mathrm{q}, L, R, P^{i}\right)$ and let $S K^{i} \leftarrow\left\{\left(s_{w, 0}^{i}, s_{w, 1}^{i}\right): w \in\{1, \ldots, \mathrm{n}\}\right\}$.

- $P_{1}$ outputs the tuple $\left(G C^{1}, S K^{1},\left\{\left(\left\langle b_{w}\right\rangle^{(1)},\left\langle\lambda_{w}\right\rangle^{(1)}, b_{w}, \lambda_{w}\right): w \in\left\{1, \ldots, \mathbf{n}_{1}\right\}\right\}\right)$.

- $P_{2}$ outputs the tuple $\left(G C^{2}, S K^{2},\left\{\left(\left\langle b_{w}\right\rangle^{(2)},\left\langle\lambda_{w}\right\rangle^{(2)}, b_{w}, \lambda_{w}\right): w \in\left\{\mathbf{n}_{1}+1, \ldots, \mathbf{n}\right\}\right\}\right)$.

${ }^{a}$ Note that we do not in fact need to create 'zero' masks for the output wires; we include this step mainly for ease of presentation.

Figure 2: Two-party distributed circuit garbling protocol. For semi-honest security use standard secret sharing for the bits; for malicious security use authenticated secret sharing.

Note that we can use this two-party distributed garbling scheme as a building block for a somewhat efficient semi-honest two-party secure computation protocol. See Appendix D for the 
detailed construction. We do not claim that this scheme is superior to existing $2 \mathrm{PC}$ protocols; however, it serves as an important building-block to our end goal of an efficient 3PC protocol.

Also note that this distributed garbling scheme can scale to more than two parties, given access to multi-party variants of the necessary functionalities. Thus, we can also achieve (semi-honest) multiparty secure computation using this approach; we leave the development of efficient instantiations of these functionalities as future work.

\subsection{Achieving Malicious Security}

The semi-honest distributed garbling scheme described in Section 3.2 can be directly adapted to work against a malicious adversary by modifying the hybrid functionalities to work in an authenticated manner; namely, we use authenticated sharings in place of standard secret sharings:

- $\mathcal{F}_{\text {const }}^{1}()$ and $\mathcal{F}_{\text {rand }}():$ The output share is authenticated.

- $\mathcal{F}_{\text {gate }}^{G}(\langle a\rangle,\langle b\rangle):$ The inputs and outputs are all authenticated sharings.

- $\mathcal{F}_{\text {oshare }}^{i}\left(\langle b\rangle, m_{0}, m_{1}\right)$ : The selection bit $b$ is an authenticated sharing.

- $\mathcal{F}_{\mathbf{s s}}^{i}(b)$ : The output is an authenticated sharing of $b$.

See Appendix A for the detailed descriptions.

We also need to define a notion of encrypting authenticated shares. Recall that for an authenticated share $\langle b\rangle=\left(\langle b\rangle^{(1)},\langle b\rangle^{(2)}\right)$, we have $\langle b\rangle^{(i)}=\left(b_{i}, t_{i}, k_{j}\right)$, where party $P_{i}$ holds $b_{i}$ and $t_{i}$ and party $P_{j}$ holds $k_{j}$. Thus, letting $K=\left(s^{1}, s^{2}\right)$, we define

$$
\operatorname{Enc}_{K}^{T}(\langle b\rangle)=\left(\operatorname{Enc}_{s^{1}, T}^{1}\left(b_{1}\left\|t_{1}\right\| k_{1}\right), \operatorname{Enc}_{s^{2}, T}^{2}\left(b_{2}\left\|t_{2}\right\| k_{2}\right)\right) .
$$

On decryption, each party's ciphertext is decrypted and the authenticity of $b_{1}$ and $b_{2}$ are verified using the (encrypted) tags and keys. Thus, when evaluating a garbled circuit, the party checks the authenticity of the share from the decrypted row of each garbled gate; if the check fails, the party aborts.

Again, we can convert this garbling scheme into a (now maliciously-secure) 2PC scheme; see Appendix D for the details. Likewise, we could also construct an MPC variant with efficient multi-party instantiations of the underlying functionalities which we leave as future work.

\section{Three-Party Computation from Cut-and-Choose}

As mentioned above, we can directly adapt the distributed garbling scheme to work over multiple parties, and thus construct a 3PC scheme; however, in this case the underlying functionalities need to support multiple parties rather than just two parties and are thus unlikely to be more efficient in practice. Thus, in this section we show how to utilize the maliciously secure two-party distributed garbling scheme from Section 3 to construct a maliciously secure three-party secure computation protocol, using almost entirely two-party constructs (the only three-party functionality needed is that of coin-tossing).

We first cover preliminary notions, such as the ideal functionalities we need, in Section 4.1. Then, in Section 4.2 we show how to adapt a combination of two existing cut-and-choose protocols [LP07, LP11 to the three-party setting. Finally, in Section 4.3 we use this "generic" protocol to show how to adapt Lindell's protocol [Lin13] (the current state-of-the-art Yao-based protocol at the time 
of writing) to the three-party setting. The cost of each of these three-party protocols is roughly eight times the computational cost of the underlying two-party protocol they are based on, and roughly sixteen times the communication cost (plus the cost of a small number of OTs per gate, which can be efficiently amortized using OT extension [IKNP03, NNOB12]), and thus we show that we can achieve efficient secure three-party computation at only a small factor of the cost of the most efficient Yao-based two-party protocol.

\subsection{Preliminaries}

Ideal Functionalities. In addition to the ideal functionalities used in the two-party distributed garbling scheme, we need the following additional (maliciously secure) functionalities:

- Three-party coin-flipping $\mathcal{F}_{\mathbf{c f}}()$ : The functionality outputs a random bit-string $\rho \leftarrow \$\{0,1\}^{s}$ to each party.

- One-out-of-two oblivious transfer $\mathcal{F}_{\mathbf{o t}}^{i, j}\left(b, m_{0}, m_{1}\right)$ : The functionality takes as input a choice bit $b$ from party $P_{i}$ and messages $m_{0}, m_{1}$ from $P_{j}$, and outputs $m_{b}$ to party $P_{i}$.

- ZKPoK of extended Diffie-Hellman tuple $\mathcal{F}_{\mathbf{z k p o k}}^{i, j}\left(a,\left(g, h_{0}, h_{1},\left\{u_{i}, v_{i}\right\}_{i}\right)\right)$ : The functionality takes as input $a$ from party $P_{i}$, and tuple $\left(g, h_{0}, h_{1},\left\{u_{i}, v_{i}\right\}_{i}\right)$ from party $P_{j}$, and outputs 1 to party $P_{j}$ if either all tuples in $\left\{\left(g, h_{0}, u_{i}, v_{i}\right)\right\}_{i}$ are Diffie-Hellman tuples with $h_{0}=g^{a}$ or all tuples in $\left\{\left(g, h_{1}, u_{i}, v_{i}\right)\right\}_{i}$ are Diffie-Hellman tuples with $h_{1}=g^{a}$, and 0 otherwise.

These can all be efficiently instantiated in a standard fashion. We can implement $\mathcal{F}_{\text {cf }}$ in the Random Oracle Model using three commitments and openings. The $\mathcal{F}_{\text {ot }}$ functionality can be instantiated using any maliciously secure OT implementation, such as the construction by Peikert et al. [PVW08]. Likewise, $\mathcal{F}_{\text {zkpok }}$ can be efficiently instantiated using existing protocols [LP11, Section B].

Distributed Garbled Circuits for Three Parties. Note that the garbling protocol $\Pi_{\mathrm{GC}}$ in Figure 2 only garbles a circuit containing inputs from two parties. We can easily adapt this to support input from a third (external) party as follows. Let $\Pi_{\mathbf{G C}}^{\prime}\left(P_{1}, P_{2}\right)$ be the same as $\Pi_{\mathbf{G C}}\left(P_{1}, P_{2}\right)$ except for the following modifications:

- All of the operations over $P_{2}$ 's input now operate over wires $w \in\left\{\mathrm{n}_{1}+1, \ldots, \mathrm{n}_{2}\right\}$.

- In Step 1, we add the following sub-step for generating shares for $P_{3}$ 's input wires:

- For $w \in\left\{\mathrm{n}_{2}+1, \ldots, \mathrm{n}\right\}$ : generate $\left\langle\lambda_{w}\right\rangle \leftarrow \mathcal{F}_{\text {rand }}$.

- In Step 4, party $P_{i}$ outputs $\left\{\left\langle\lambda_{w}\right\rangle^{(i)}: w \in\left\{\mathrm{n}_{2}+1, \ldots, \mathrm{n}\right\}\right\}$ in addition to his normal outputs.

\subsection{Achieving Malicious Security for Three Parties}

Note that our two-party distributed garbling scheme has the property that if at most one of the two parties is corrupt, the garbling of circuit $C$ either correctly evaluates $C$ on $P_{1}$ 's and $P_{2}$ 's inputs, or causes the evaluator to abort. That is, a malicious party cannot "alter" the garbling to evaluate some circuit other than $C$. Now, if both $P_{1}$ and $P_{2}$ are corrupt, they can of course garble an arbitrary circuit. This suggests the following approach to three-party computation: If either $P_{1}$ or $P_{2}$ are honest, we need only construct a single garbled circuit, which is sent to $P_{3}$ to be evaluated. To cover the case where both $P_{1}$ and $P_{2}$ are corrupt, we use cut-and-choose to prevent $P_{3}$ from evaluating a maliciously constructed circuit. In what follows, we utilize existing cut-and-choose protocols from the literature [LP07, LP11], and "plug in" our distributed garbling scheme as necessary. Thus, 
security mostly follows from the security proofs of the underlying cut-and-choose protocols. In Section 4.3, we show how we can use this protocol in an adaptation of Lindell's protocol [Lin13] to the three-party setting.

The basic intuition for security is as follows. Cut-and-choose is used to prevent $P_{3}$ from evaluating maliciously constructed circuits when both $P_{1}$ and $P_{2}$ are malicious. For the case where either $P_{1}$ or $P_{2}$ is honest, $\Pi_{\mathbf{G C}}^{\prime}\left(P_{1}, P_{2}\right)$ assures us that the garbled circuit constructed between $P_{1}$ and $P_{2}$ is either correctly constructed or causes $P_{3}$ to abort (independent of any party's input).

Protocol Description. We assume the reader is familiar with the cut-and-choose technique; here we briefly discuss the main technical challenges that result from a naïve application of cut-and-choose and how we address them.

- Input Inconsistency. The use of cut-and-choose produces multiple garbled circuits to be evaluated by $P_{3}$. The idea with this attack is that a given party (either $P_{1}$ or $P_{2}$ in the three-party case) can give inconsistent sub-keys in each of these circuits such that $P_{3}$ ends up evaluating different inputs for $P_{1} / P_{2}$ instead of consistent inputs across all garbled circuits. This is a well-known attack, and there are multiple solutions in the two-party setting. Here, we use the Diffie-Hellman pseudorandom synthesizer trick [MF06, LP11] and adapt it in a straightforward manner to the three-party setting.

- Selective Failure. This attack arises any time the parties execute an OT to send the sub-keys for $P_{3}$ 's input. Note that if the sender in the OT (either party $P_{1}$ or $P_{2}$ ) inputs one valid label and one invalid label, he can learn a bit of $P_{3}$ 's input by learning whether the garbled circuit evaluation fails or not. We circumvent this problem by directly applying the "XOR-tree" approach [LP07, Woo07].

We now give a high-level description of our protocol.

1. The parties first replace the input circuit $C^{0}$ with a circuit $C$, where the only difference is each of $P_{3}$ 's input wires is replaced by an XOR of $s$ new input wires, preventing either party $P_{1}$ or $P_{2}$ from launching a selective failure attack on $P_{3}$ 's input choices.

2. $P_{1}$ and $P_{2}$ generate the required commitments needed for input consistency, as is done in the protocol of Lindell and Pinkas [LP11].

3. $P_{1}$ and $P_{2}$ construct $s$ garbled circuits using $\Pi_{\mathbf{G C}}^{\prime}$ and the input sub-keys generated as is done in the protocol of Lindell and Pinkas [LP11].

4. $P_{1}$ and $P_{2}$ compute authenticated sharings (between each other; $P_{3}$ is not involved here) of their input bits.

5. $P_{1}$ and $P_{2}$ both run (separately) an OT protocol with $P_{3}$ for each of $P_{3}$ 's input wires, where $P_{1} / P_{2}$ input their sub-keys and $P_{3}$ chooses based on his input. (Note that any cheating by $P_{1} / P_{2}$ here will be caught with high-probability by the cut-and-choose step below.) Thus, $P_{3}$ now has keys for each of his input bits.

6. $P_{1}$ and $P_{2}$ send the (distributed) garbled circuits, along with the input consistency commitments, to $P_{3}$.

7. All three parties run a coin-tossing protocol to determine which circuits for $P_{3}$ to open and which to evaluate. 
8. For the evaluation circuits, $P_{1}$ and $P_{2}$ send the sub-keys and selector bits for their inputs to $P_{3}$. Note that we need to be careful in this step, as we need to enforce that, for example, $P_{1}$ uses the same input as was shared in Step 2 above. This is accomplished as follows. Recall that $P_{1}$ and $P_{2}$ have sharings of each other's inputs and mask bits, all of which are authenticated. Thus, $P_{1}$ can send the (authenticated) share of her masked input to $P_{2}$, who can verify its authenticity, and thus reconstruct the masked input bit using his own share. This allows an honest $P_{2}$ to send the correct sub-key (correct in the sense that it corresponds to $P_{1}$ 's input shared in Step 2) to $P_{3}$, even with a malicious $P_{1}$.

9. For the check circuits, $P_{1}$ and $P_{2}$ send the required information for $P_{3}$ to decrypt the check circuits and verify correctness. If any of these check circuits are incorrectly constructed, $P_{3}$ aborts; otherwise, he has high confidence that the majority of the evaluation circuits are correctly constructed.

10. For the evaluation circuits, $P_{3}$ checks for input consistency against the sub-keys sent by $P_{1}$ and $P_{2}$ in Step 8 using a zero-knowledge proof-of-knowledge protocol [LP11], aborting on any inconsistency.

11. Finally, $P_{3}$ evaluates the evaluation circuits, outputting the majority over the circuits' output.

See below for the full protocol description.

\section{Protocol $\Pi_{3 \mathrm{PC}}^{m}\left(P_{1}, P_{2}, P_{3}\right)$}

Auxiliary Inputs: Security parameter $k$, statistical security parameter $s$, circuit $C^{0}$, cyclic group $\mathbb{G}$ with (prime) order $q$ and generator $g$, and randomness extractor $H$.

Inputs: For $w \in\left\{1, \ldots, \mathrm{n}_{1}\right\}, P_{1}$ has inputs $b_{w} ;$ for $w \in\left\{\mathrm{n}_{1}+1, \ldots, \mathrm{n}_{2}\right\}, P_{1}$ has inputs $b_{w} ;$ for $w \in\left\{\mathrm{n}_{2}+1, \ldots, \mathbf{n}\right\}$, $P_{3}$ has inputs $b_{w}$.

1. Each party replaces $C^{0}$ with a circuit $C$ where each of $P_{3}$ 's input wires is replaced by an exclusive-or of $s$ new input wires. We let $(\mathrm{n}, \mathrm{m}, \mathrm{q}, L, R, G) \leftarrow C$, and denote $P_{3}$ 's new inputs by $\hat{b}_{w}$.

2. For $w \in\left\{1, \ldots, \mathrm{n}_{1}\right\}: P_{1}$ generates $a_{w, 0}^{1}, a_{w, 1}^{1} \leftarrow \$ \mathbb{Z}_{q}$ and constructs set $\left\{\left(w, 0, g^{a_{w, 0}^{1}}\right),\left(w, 1, g^{a_{w, 1}^{1}}\right)\right\}$.

For $w \in\left\{\mathrm{n}_{1}+1, \ldots, \mathrm{n}_{2}\right\}: P_{2}$ generates $a_{w, 0}^{2}, a_{w, 1}^{2} \leftarrow \$ \mathbb{Z}_{q}$ and constructs set $\left\{\left(w, 0, g^{a_{w, 0}^{2}}\right),\left(w, 1, g^{a_{w, 1}^{2}}\right)\right\}$.

For $j \in\{1, \ldots, s\}: P_{i}$, for $i \in\{1,2\}$, generates $r_{j}^{i} \leftarrow \$ \mathbb{Z}_{q}$ and constructs set $\left\{\left(j, g^{r_{j}^{i}}\right)\right\}$.

For $j \in\{1, \ldots, s\}: P_{1}$ and $P_{2}$ run up to Step 2 (Generate sub-keys") of $\Pi_{\mathbf{G C}}^{3}\left(P_{1}, P_{2}\right)$, where the parties do the following in the $j$ th iteration:

- For $w \in\left\{1, \ldots, \mathrm{n}_{1}\right\}: P_{1}$ generates sub-keys $s_{w, b \oplus \lambda_{w, j}, j}^{1} \leftarrow H\left(g^{a_{w, b}^{1} \cdot r_{j}^{1}}\right)$ for $b \in\{0,1\}$.

- For $w \in\left\{\mathbf{n}_{1}+1, \ldots, \mathbf{n}_{2}\right\}: P_{2}$ generates sub-keys $s_{w, b \oplus \lambda_{w, j}, j}^{2} \leftarrow H\left(g^{a_{w, b}^{2} \cdot r_{j}^{2}}\right)$ for $b \in\{0,1\}$.

- All other sub-keys are generated in the normal fashion.

3. For $j \in\{1, \ldots, s\}: P_{1}$ and $P_{2}$ continue their executions of $\Pi_{\mathbf{G C}}^{3}\left(P_{1}, P_{2}\right)$, producing garbled circuit $G C_{j}$.

4. For $w \in\left\{1, \ldots, \mathrm{n}_{1}\right\}: P_{1}$ and $P_{2}$ compute $\left\langle b_{w}\right\rangle \leftarrow \mathcal{F}_{\text {ss }}^{1}\left(b_{w}\right)$.

For $w \in\left\{\mathbf{n}_{1}+1, \ldots, \mathbf{n}_{2}\right\}: P_{1}$ and $P_{2}$ compute $\left\langle b_{w}\right\rangle \leftarrow \mathcal{F}_{\mathbf{s s}}^{2}\left(b_{w}\right)$.

5. For $j \in\{1, \ldots, s\}$ and $w \in\left\{\mathrm{n}_{2}+1, \ldots, \mathrm{n}\right\}: P_{1}$ and $P_{2}$ exchange $\left\langle\lambda_{w, j}\right\rangle$ with each other, reconstructing $\lambda_{w, j}$ locally. Both $P_{1}$ and $P_{2}$ send $\lambda_{w, j}$ to $P_{3}$.

For $w \in\left\{\mathrm{n}_{2}+1, \ldots, \mathrm{n}\right\}: P_{i}$, for $i \in\{1,2\}$, and $P_{3}$ run $\mathcal{F}_{\mathbf{o t}}$, with $P_{i}$ as the sender inputting $\left(\left\{s_{w, \lambda_{w, j}, j}^{i}\right\}_{j \in\{1, \ldots, s\}},\left\{s_{w, \lambda_{w, j} \oplus 1, j}^{i}\right\}_{j \in\{1, \ldots, s\}}\right)$ and $P_{3}$ as the receiver inputting $\hat{b}_{w}$.

6. $P_{i}$, for $i \in\{1,2\}$, sends the sets constructed in Step 2 along with the garbled circuit $\left\{G C_{j}^{i}\right\}_{i=1}^{s}$, to $P_{3}$. 
7. The parties compute $\rho \leftarrow \mathcal{F}_{\text {cf }}$. Let $\mathcal{C C}=\left\{i: \rho_{i}=1\right\}$, and let $\mathcal{E C}=\{1, \ldots, s\} \backslash \mathcal{C C}$.

8. For $j \in \mathcal{E C}$ :

- For $w \in\left\{1, \ldots, \mathbf{n}_{1}\right\}: P_{1}$ sends $\left\langle b_{w}\right\rangle^{(1)} \oplus\left\langle\lambda_{w, j}\right\rangle^{(1)}$ to $P_{2}$, who reconstructs $b_{w} \oplus \lambda_{w, j}$ locally. $P_{1}$ sends $\left(s_{w, b_{w} \oplus \lambda_{w, j}, j}^{1}, b_{w} \oplus \lambda_{w, j}\right)$ to $P_{3}$, and $P_{2}$ sends $\left(s_{w, b_{w} \oplus \lambda_{w, j}, j}^{2}, b_{w} \oplus \lambda_{w, j}\right)$ to $P_{3}$.

- For $w \in\left\{\mathbf{n}_{1}+1, \ldots, \mathbf{n}\right\}: P_{2}$ sends $\left\langle b_{w}\right\rangle^{(2)} \oplus\left\langle\lambda_{w, j}\right\rangle^{(2)}$ to $P_{1}$, who reconstructs $b_{w} \oplus \lambda_{w, j}$ locally. $P_{1}$ sends $\left(s_{w, b_{w} \oplus \lambda_{w, j}, j}^{1}, b_{w} \oplus \lambda_{w, j}\right)$ to $P_{3}$, and $P_{2}$ sends $\left(s_{w, b_{w} \oplus \lambda_{w, j}, j}^{2}, b_{w} \oplus \lambda_{w, j}\right)$ to $P_{3}$.

9. For $j \in \mathcal{C C}$ :

- $\quad P_{i}$, for $i \in\{1,2\}$, does the following:

- Sends $r_{j}^{i}$ to $P_{3}$, and $P_{3}$ checks that these values are consistent with the pairs $\left\{\left(j, g^{r_{j}^{i}}\right)\right\}$ sent before.

- For $w \in\{1, \ldots, \mathbf{n}\}$ : Sends sub-keys $s_{w, 0, j}^{i}$ and $s_{w, 1, j}^{i}$, mask bit share $\lambda_{w, j}^{(i)}$, and the keys to the authenticated bits to $P_{3}$.

- Given the above information, $P_{3}$ reconstructs all input labels and verifies they match with those labels sent previously. Also, using said labels, $P_{3}$ verifies that the garbled circuit is correctly constructed.

10. For $j \in \mathcal{E C}$ :

- For $w \in\left\{1, \ldots, \mathbf{n}_{1}\right\}: P_{1}$ sends $g^{a_{w, b_{w}}^{1} \cdot r_{j}^{1}}$ to $P_{3}$, who computes $s_{w, b_{w} \oplus \lambda_{w, j}, j}^{1} \leftarrow H\left(g^{a_{w, b_{w}}^{1} \cdot r_{j}^{1}}\right)$.

- For $w \in\left\{\mathrm{n}_{1}+1, \ldots, \mathrm{n}_{2}\right\}: P_{2}$ sends $g^{a_{w, b_{w}}^{2} \cdot r_{j}^{2}}$ to $P_{3}$, who computes $s_{w, b_{w} \oplus \lambda_{w, j}, j}^{2} \leftarrow H\left(g^{a_{w, b}^{2} \cdot r_{j}^{2}}\right)$.

For $w \in\left\{1, \ldots, \mathbf{n}_{1}\right\}: P_{1}$ and $P_{3}$ run $\mathcal{F}_{\mathbf{z k p o k}}$, with $P_{1}$ as the prover inputting $a_{w, b_{w}}^{1}$ and $P_{3}$ as the verifier inputting $\left(g, g^{a_{w, 0}^{1}}, g^{a_{w, 1}^{1}},\left\{\left(g^{r_{j}^{1}}, g^{a_{w, b}^{1}} \cdot r_{j}^{1}\right)\right\}_{j \in \mathcal{E C}}\right)$.

For $w \in\left\{\mathrm{n}_{1}+1, \ldots, \mathrm{n}_{2}\right\}: P_{2}$ and $P_{3}$ run $\mathcal{F}_{\text {zkpok }}$, with $P_{2}$ as the prover inputting $a_{w, b_{w}}^{2}$ and $P_{3}$ as the verifier inputting $\left(g, g^{a_{w, 0}^{2}}, g^{a_{w, 1}^{2}},\left\{\left(g^{r_{j}^{2}}, g^{a_{w, b}^{2} \cdot r_{j}^{2}}\right)\right\}_{j \in \mathcal{E C}}\right)$.

11. For $j \in \mathcal{E C}: P_{3}$ evaluates circuit $G C_{j}$ using $\left\{\left(s_{w, b_{w} \oplus \lambda_{w, j}, j}^{1}, s_{w, b_{w} \oplus \lambda_{w, j}, j}^{2}, b_{w} \oplus \lambda_{w, j}\right)\right\}_{w \in\{1, \ldots, \mathrm{n}\}}$ as inputs. $P_{3}$ outputs the majority output over the evaluated circuits.

Theorem 1. Let $C$ be an arbitrary polynomial-size circuit and let $\mathbb{G}$ be a cyclic group with prime order. Given access to ideal functionalities $\mathcal{F}_{\text {const }}, \mathcal{F}_{\text {gate }}, \mathcal{F}_{\text {oshare }}, \mathcal{F}_{\text {ot }}, \mathcal{F}_{\text {rand }}$, and $\mathcal{F}_{\text {ss }}$, and assuming that the decisional Diffie-Hellman problem is hard in $\mathbb{G}$, then $\Pi_{\mathbf{3} \mathbf{P C}}^{m}\left(P_{1}, P_{2}, P_{3}\right)$ securely computes the circuit $C$ in the presence of an adversary corrupting an arbitrary number of parties.

Proof. See Appendix E,

\subsection{Adapting Lindell's Protocol to the Three-Party Setting}

The $3 \mathrm{PC}$ protocol described above has a replication factor of roughly $3 \times$; namely, for statistical security parameter $s$, the actual probability of cheating is roughly $2^{-0.32 s}$ [LP11]. Thus, for a desired error probability of $2^{-40}$ a total of 128 circuits need to be garbled. Recently, Lindell [Lin13] showed a beautiful construction which removes this replication factor in the two-party setting; that is, for a cheating probability of $2^{-s}$ the sender needs to garble only $s$ circuits. In this section we show how to adapt this protocol to the three-party setting.

Lindell's construction works in two phases. In the first phase, the parties do a standard cutand-choose, with $P_{1}$ constructing $s$ circuits (for error probability $2^{-s}$ ) and $P_{2}$ opening half of them. If, during evaluation, $P_{2}$ finds that two or more circuits have conflicting outputs, it stores these conflicting outputs as a "proof-of-cheating" $\phi$. In the second phase, the parties run a circuit which 
takes as input from $P_{1}$ her original input $x$, and from $P_{2}$ the "proof-of-cheating" $\phi$. If $\phi$ is a valid proof, then the circuit reveals $x$ to $P_{2}$, who can then compute the output himself; otherwise $P_{2}$ gets no output. Thus, this second phase enforces that if $P_{1}$ cheated in the cut-and-choose then $P_{2}$ learns $P_{1}$ 's input.

To adapt this to the three-party setting, we proceed as follows. For the circuit in the first phase, we essentially just run $\Pi_{3 \mathbf{P C}}^{m}\left(P_{1}, P_{2}, P_{3}\right)$, with the same tweaks as are used by Lindell [Lin13] (namely, the use of encoded output translation tables and doing circuit evaluation before circuit checking).

For the second phase circuit, we run into some issues, due to Lindell's scheme being inherently a "two-party" approach. Recall that this circuit is constructed in such a way that if $P_{2}$ receives any conflicting outputs when evaluating, he inputs these outputs as a "proof-of-cheating" in order to reveal $P_{1}$ 's input. At first glance, it appears this technique would not work in the three-party setting because in that case $P_{3}$ needs to learn both $P_{1}$ 's and $P_{2}$ 's inputs to reconstruct the output; however, it could be the case that only one of these two parties is cheating. Recall, however, that our distributed garbling scheme enforces that as long as one of the two parties is honest, the garbled circuits are "correct" in the sense that they either correctly compute the desired circuit are cause a failure independent of any party's input. Thus, $P_{3}$ only finds mismatched outputs in the case where both $P_{1}$ and $P_{2}$ cheat, making it okay at this point to reveal both those parties' inputs in the second phase circuit.

Another issue arises in how this circuit is constructed. In Lindell's two-party scheme, $P_{1}$ hardwires the output keys into the circuit. In a naïve adaptation to the three-party setting, both $P_{1}$ and $P_{2}$ would need to hardwire their output $s u b$-keys into the circuit. However, this would allow each party to learn the others' sub-keys for the output, which leads to the following attack by a colluding $P_{1}$ and $P_{3}$ : During the construction of the second phase circuit, $P_{1}$ learns $P_{2}$ 's output sub-keys, and he sends these, as well as his own output sub-keys, to $P_{3}$. Now, when $P_{3}$ evaluates the circuit, he can input conflicting outputs as his "proof-of-cheating" because he knows all of the outputs keys, thus allowing $P_{1}$ and $P_{3}$ to learn $P_{2}$ 's input. We can fix this by having the output sub-keys of $P_{1}$ and $P_{2}$ be inputs to the circuit, rather than hardcoded. However, this raises another issue, as $P_{3}$ cannot verify that the sub-keys input by $P_{1}$ and $P_{2}$ are the correct ones. Thus, we modify the circuit to output these sub-keys in the clear, allowing $P_{3}$ to do this check.

See below for the full protocol description.

$$
\text { Protocol } \Pi_{3 \mathrm{PC}}^{m-\text { lindell }}\left(P_{1}, P_{2}, P_{3}\right)
$$

Auxiliary Inputs: Security parameter $k$, statistical security parameter $s$, circuit $C^{0}$, cyclic group $\mathbb{G}$ with (prime) order $q$ and generator $g$, and randomness extractor $H$.

Inputs: For $w \in\left\{1, \ldots, \mathrm{n}_{1}\right\}, P_{1}$ has inputs $b_{w}$; for $w \in\left\{\mathbf{n}_{1}+1, \ldots, \mathrm{n}_{2}\right\}, P_{2}$ has inputs $b_{w} ;$ for $w \in\left\{\mathbf{n}_{2}+1, \ldots, \mathbf{n}\right\}$, $P_{3}$ has inputs $b_{w}$.

1. Each party replaces $C^{0}$ with a circuit $C$ where each of $P_{3}$ 's input wires is replaced by an exclusive-or of $s$ new input wires. We let $(\mathrm{n}, \mathrm{m}, \mathrm{q}, L, R, G) \leftarrow C$, and denote $P_{3}$ 's new inputs by $\hat{b}_{w}$.

2. For $w \in\left\{1, \ldots, \mathrm{n}_{1}\right\}: P_{1}$ generates $a_{w, 0}^{1}, a_{w, 1}^{1} \leftarrow \$ \mathbb{Z}_{q}$ and constructs set $\left\{\left(w, 0, g^{a_{w}^{1}, 0}\right),\left(w, 1, g^{a_{w, 1}^{1}}\right)\right\}$.

For $w \in\left\{\mathrm{n}_{1}+1, \ldots, \mathrm{n}_{2}\right\}: P_{2}$ generates $a_{w, 0}^{2}, a_{w, 1}^{2} \leftarrow \$ \mathbb{Z}_{q}$ and constructs set $\left\{\left(w, 0, g^{a_{w, 0}^{2}}\right),\left(w, 1, g^{a_{w, 1}^{2}}\right)\right\}$.

For $w \in\{\mathrm{n}+\mathrm{q}-\mathrm{m}+1, \ldots, \mathrm{n}+\mathrm{q}\}: P_{i}$, for $i \in\{1,2\}$, generates $o_{w, 0}^{i}, o_{w, 1}^{i} \leftarrow \$\{0,1\}^{k}$.

For $j \in\{1, \ldots, s\}: P_{i}$, for $i \in\{1,2\}$, generates $r_{j}^{i} \leftarrow \$ \mathbb{Z}_{q}$ and constructs set $\left\{\left(j, g^{r_{j}^{i}}\right)\right\}$. 
For $j \in\{1, \ldots, s\}: P_{1}$ and $P_{2}$ run up to Step 2 ("Generate sub-keys") of $\Pi_{\mathbf{G C}}^{\prime}\left(P_{1}, P_{2}\right)$, where the parties do the following:

- For $w \in\left\{1, \ldots, \mathrm{n}_{1}\right\}: P_{1}$ generates sub-keys $s_{w, b \oplus \lambda_{w, j}, j}^{1} \leftarrow H\left(g^{a_{w, b}^{1} \cdot r_{j}^{1}}\right)$ for $b \in\{0,1\}$.

- For $w \in\left\{\mathrm{n}_{1}+1, \ldots, \mathrm{n}_{2}\right\}: P_{2}$ generates sub-keys $s_{w, b \oplus \lambda_{w, j}, j}^{2} \leftarrow H\left(g^{a_{w, b}^{2} \cdot r_{j}^{2}}\right)$ for $b \in\{0,1\}$.

- For $w \in\{\mathbf{n}+\mathbf{q}-\mathbf{m}+1, \ldots, \mathbf{n}+\mathbf{q}\}: P_{i}$ sets $s_{w, b \oplus \lambda, j, j}^{i} \leftarrow o_{w, b}^{i}$.

- All other sub-keys are generated in the normal fashion.

3. For $j \in\{1, \ldots, s\}: P_{1}$ and $P_{2}$ continue their executions of $\Pi_{\mathbf{G C}}^{\prime}\left(P_{1}, P_{2}\right)$, producing (distributed) garbled circuit $G C_{j}:=\left(G C_{j}^{1}, G C_{j}^{2}\right)$.

4. For $w \in\left\{1, \ldots, \mathbf{n}_{1}\right\}: P_{1}$ and $P_{2}$ compute $\left\langle b_{w}\right\rangle \leftarrow \mathcal{F}_{\mathbf{s s}}^{1}\left(b_{w}\right)$.

For $w \in\left\{\mathrm{n}_{1}+1, \ldots, \mathrm{n}_{2}\right\}: P_{1}$ and $P_{2}$ compute $\left\langle b_{w}\right\rangle \leftarrow \mathcal{F}_{\mathbf{s s}}^{2}\left(b_{w}\right)$.

5. For $j \in\{1, \ldots, s\}$ and $w \in\left\{\mathbf{n}_{2}+1, \ldots, \mathbf{n}\right\}: P_{1}$ and $P_{2}$ exchange $\left\langle\lambda_{w, j}\right\rangle$ with each other, reconstructing $\lambda_{w, j}$ locally. Both $P_{1}$ and $P_{2}$ send $\lambda_{w, j}$ to $P_{3}$.

For $w \in\left\{\mathrm{n}_{2}+1, \ldots, \mathbf{n}\right\}: P_{i}$, for $i \in\{1,2\}$, and $P_{3}$ run $\mathcal{F}_{\mathbf{o t}}$, with $P_{i}$ as the sender inputting $\left(\left\{s_{w, \lambda_{w, j}, j}^{i}\right\}_{j \in\{1, \ldots, s\}},\left\{s_{w, \lambda_{w, j} \oplus 1, j}^{i}\right\}_{j \in\{1, \ldots, s\}}\right)$ and $P_{3}$ as the receiver inputting $\hat{b}_{w}$.

6. For $i \in\{1,2\}: P_{i}$ sends the sets constructed in Step 2, along with the garbled circuit $\left\{G C_{j}^{i}\right\}_{j=1}^{s}$, to $P_{3}$. In addition, $P_{i}$ sends the encoded output translation table $\left\{\left(H\left(o_{w, 0}^{i}\right), H\left(o_{w, 1}^{i}\right)\right)\right\}_{w=\mathrm{n}+\mathrm{q}-\mathrm{m}+1}^{\mathrm{n}+\mathrm{q}}$ to $P_{3}$.

7. The parties compute $\rho \leftarrow \mathcal{F}_{\text {cf }}$. Let $\mathcal{C C}=\left\{i: \rho_{i}=1\right\}$, and let $\mathcal{E C}=\{1, \ldots, s\} \backslash \mathcal{C C}$.

8. For $j \in \mathcal{E C}$ (the evaluation circuits):

- For $w \in\left\{1, \ldots, \mathbf{n}_{1}\right\}: P_{1}$ sends $\left\langle b_{w}\right\rangle^{(1)} \oplus\left\langle\lambda_{w, j}\right\rangle^{(1)}$ to $P_{2}$, who reconstructs $b_{w} \oplus \lambda_{w, j}$ locally. $P_{1}$ sends $\left(s_{w, b_{w} \oplus \lambda_{w, j}, j}^{1}, b_{w} \oplus \lambda_{w, j}\right)$ to $P_{3}$, and $P_{2}$ sends $\left(s_{w, b_{w} \oplus \lambda_{w, j}, j}^{2}, b_{w} \oplus \lambda_{w, j}\right)$ to $P_{3}$.

- For $w \in\left\{\mathbf{n}_{1}+1, \ldots, \mathbf{n}\right\}: P_{2}$ sends $\left\langle b_{w}\right\rangle^{(2)} \oplus\left\langle\lambda_{w, j}\right\rangle^{(2)}$ to $P_{1}$, who reconstructs $b_{w} \oplus \lambda_{w, j}$ locally. $P_{1}$ sends $\left(s_{w, b_{w} \oplus \lambda_{w, j}, j}^{1}, b_{w} \oplus \lambda_{w, j}\right)$ to $P_{3}$, and $P_{2}$ sends $\left(s_{w, b_{w} \oplus \lambda_{w, j}, j}^{2}, b_{w} \oplus \lambda_{w, j}\right)$ to $P_{3}$.

- For $w \in\left\{1, \ldots, \mathrm{n}_{1}\right\}: P_{1}$ sends $k_{w, j}^{1}:=g^{a_{w, b_{w}}^{1} \cdot r_{j}^{1}}$ to $P_{3}$, who computes $s_{w, b_{w} \oplus \lambda_{w, j}, j}^{1} \leftarrow H\left(g^{a_{w, b_{w}}^{1} \cdot r_{j}^{1}}\right)$.

- For $w \in\left\{\mathrm{n}_{1}+1, \ldots, \mathrm{n}_{2}\right\}: P_{2}$ sends $k_{w, j}^{2}:=g^{a_{w, b}^{2}} \cdot r_{j}^{2}$ to $P_{3}$, who computes $s_{w, b_{w} \oplus \lambda_{w, j}, j}^{2} \leftarrow H\left(g^{a_{w, b}^{2}} \cdot r_{j}^{2}\right)$.

- $P_{3}$ evaluates circuit $G C_{j}$ using $\left\{\left(s_{w, b_{w} \oplus \lambda_{w, j}, j}^{1}, s_{w, b_{w} \oplus \lambda_{w, j}, j}^{2}, b_{w} \oplus \lambda_{w, j}\right)\right\}_{w \in\{1, \ldots, \mathrm{n}\}}$ as inputs.

$P_{3}$ uses the encoded output translation tables sent in Step 6 to check if he received exactly one valid output value for each output wire. If not, he stores these outputs as $o_{0}^{j}$ and $o_{1}^{j}$ and continues.

9. $\quad P_{1}$ and $P_{2}$ construct a circuit $C^{\prime}$ as follows:

- $\quad P_{1}$ inputs string $x \in\{0,1\}^{\mathrm{n}_{1}}$ and strings $o_{w, 0}^{i}, o_{w, 1}^{i} \in\{0,1\}^{k}$, for $w \in\{\mathbf{n}+\mathbf{q}-\mathbf{m}+1, \ldots, \mathbf{n}+\mathbf{q}\}$.

- $\quad P_{1}$ inputs string $y \in\{0,1\}^{\mathrm{n}_{2}-\mathrm{n}_{1}}$ and strings $o_{w, 0}^{2}, o_{w, 1}^{2} \in\{0,1\}^{k}$, for $w \in\{\mathrm{n}+\mathrm{q}-\mathrm{m}+1, \ldots, \mathrm{n}+\mathrm{q}\}$.

- $P_{3}$ inputs $o_{0}, o_{1} \in\{0,1\}^{k}$.

- If there exists some $j$ such that $o_{j, 0}^{1} \| o_{j, 0}^{2}=o_{0}^{j}$ and $o_{j, 1}^{1} \| o_{j, 1}^{2}=o_{1}^{j}$, then $P_{3}$ 's output is $x \| y$; otherwise $P_{3}$ receives no output.

- The circuit also outputs the values $\left\{o_{w, 0}^{1}, o_{w, 1}^{1}, o_{w, 0}^{2}, o_{w, 1}^{2}\right\}_{w=\mathrm{n}+\mathrm{q}-\mathrm{m}+1}^{\mathrm{n}+\mathrm{q}}$ input by parties $P_{1}$ and $P_{2}$ above.

The parties run $\Pi_{3 \mathbf{P C}}^{m}\left(P_{1}, P_{2}, P_{3}\right)$ on circuit $C^{\prime}$ as follows:

- $\quad P_{1}$ inputs her input $x=b_{1} \ldots b_{\mathrm{n}_{1}} ; P_{2}$ inputs his input $y=b_{\mathrm{n}_{1}+1} \ldots b_{\mathrm{n}_{2}}$.

- If $P_{3}$ received two conflicting outputs $o_{0}^{1} \| o_{0}^{2}$ and $o_{1}^{1} \| o_{1}^{2}$ for some circuit $j \in\{1, \ldots, s\}$ in Step 8 then he inputs these values; otherwise he inputs garbage.

- The garbled circuit uses the same $a_{w, 0}^{1}, a_{w, 1}^{1}, a_{w, 0}^{2}, a_{w, 1}^{2}$ values as in Step 2

$P_{3}$ verifies that the values $\left\{o_{w, 0}^{1}, o_{w, 1}^{1}, o_{w, 0}^{2}, o_{w, 1}^{2}\right\}_{w=\mathrm{n}+\mathrm{q}-\mathrm{m}+1}^{\mathrm{n}+\mathrm{q}}$ output by $C^{\prime}$ match those in the encoded output translation tables sent in Step 6

10. For $j \in \mathcal{C C}$ (the check circuits):

- $\quad P_{i}$, for $i \in\{1,2\}$, does the following:

- Sends $r_{j}^{i}$ to $P_{3}$, and $P_{3}$ checks that these values are consistent with the pairs $\left\{\left(j, g^{r_{j}^{i}}\right)\right\}$ sent before. 
- For $w \in\{1, \ldots, \mathbf{n}\}$ : Sends sub-keys $s_{w, 0, j}^{i}$ and $s_{w, 1, j}^{i}$, mask bit share $\lambda_{w, j}^{(i)}$, and the keys to the authenticated bits to $P_{3}$.

- Given the above information, $P_{3}$ reconstructs all input labels and verifies they match with those labels sent previously. Also, using said labels, $P_{3}$ verifies that the garbled circuit is correctly constructed.

11. For the cut-and-choose computation from Step 9, let $\widehat{\mathcal{E E}}$ be the check circuits, let $\widehat{r}_{j}^{i}$ be analogous to the $r_{j}^{i}$ values from Step 2, and let $\widehat{k}_{w, j}^{i}$ be analogous to the $k_{w, j}^{i}$ from Step 6 .

For $w \in\left\{1, \ldots, \mathrm{n}_{1}\right\}: P_{1}$ and $P_{3}$ run a zero-knowledge proof-of-knowledge, with $P_{1}$ proving that there exists some $b_{w} \in\{0,1\}$ such that for every $j \in \mathcal{E E}$ and for every $j^{\prime} \in \widehat{\mathcal{E E}}, k_{w, j}^{1}=g^{a_{w, b}^{1} \cdot r_{j}^{1}}$ and $\widehat{k}_{w, j^{\prime}}^{1}=g^{a_{w, b_{w}}^{1} \cdot \widehat{r}_{j^{\prime}}^{1}}$. For $w \in\left\{\mathrm{n}_{1}+1, \ldots, \mathrm{n}_{2}\right\}: P_{2}$ and $P_{3}$ run a zero-knowledge proof-of-knowledge, with $P_{2}$ proving that there exists some $b_{w} \in\{0,1\}$ such that for every $j \in \mathcal{E E}$ and for every $j^{\prime} \in \widehat{\mathcal{E E}}, k_{w, j}^{2}=g^{a_{w}^{2} b_{w} \cdot r_{j}^{2}}$ and $\widehat{k}_{w, j^{\prime}}^{2}=g^{a_{w, b_{w}}^{2} \cdot \widehat{r}_{j^{\prime}}^{2}}$.

12. $P_{3}$ either outputs the output received in the evaluation circuits, or, if $P_{3}$ received any inconsistent inputs in Step 8 , then it locally computes $f(x, y, z)$, where $x$ and $y$ are the inputs $P_{3}$ received in Step 9 and $z$ is $P_{3}$ 's own input.

Theorem 2. Let $C$ be an arbitrary polynomial-size circuit and let $\mathbb{G}$ be a cyclic group with prime order. Given access to ideal functionalities $\mathcal{F}_{\text {const }}, \mathcal{F}_{\text {gate }}, \mathcal{F}_{\text {oshare }}, \mathcal{F}_{\text {ot }}, \mathcal{F}_{\text {rand }}$, and $\mathcal{F}_{\text {ss }}$, and assuming that the decisional Diffie-Hellman problem is hard in $\mathbb{G}$, then $\Pi_{\mathbf{3} \mathbf{P C}}^{m-\text { lindell }}\left(P_{1}, P_{2}, P_{3}\right)$ securely computes the circuit $C$ in the presence of an adversary corrupting an arbitrary number of parties.

Proof. See Appendix F.

\subsection{Efficiency}

We now argue why our 3PC protocol is roughly eight times as expensive in terms of computation as the underlying $2 \mathrm{PC}$ protocol we utilize, and roughly sixteen times as expensive in terms of communication. Both protocols are very similar to the underlying $2 \mathrm{PC}$ protocol they are based on; the major changes in terms of computation cost are that (1) the cost of encrypting a single row increases due to the use of the distributed encryption scheme, and (2) $P_{3}$ needs to do twice the work (due to needing to communicate with both $P_{1}$ and $P_{2}$ ) as compared to the evaluator in the underlying 2PC protocol. Indeed, it takes about eight PRF calls (where one PRF call equals outputting $k$ bits) to encrypt a single row of the garbled circuit, and thus the cost and size of a garbled circuit increases by a factor of eight. The cost for $P_{1}$ and $P_{2}$ to distributively garble a circuit is a small number of OTs per gate, and this can be amortized using OT extension techniques [KNP03.

In terms of communication cost, both $P_{1}$ and $P_{2}$ need to send their half of the distributed garbled circuit to $P_{3}$, and the communication cost of actually constructing a distributed garbled circuit is roughly the cost of a standard garbled circuit. Since each garbled circuit is eight times larger than in the underlying $2 \mathrm{PC}$ protocol, we find that the overall communication size increases by approximately sixteen.

\section{Efficient Instantiations of Necessary Functionalities}

In this section, we show how to efficiently instantiate the calls to the two-party functionalities used in our protocols. 
Semi-Honest Setting. The hybrids for our semi-honest protocol can be instantiated in the standard fashion, see Appendix A. For instantiating $\mathcal{F}_{\text {oshare }}$, we need a single call to $\mathcal{F}_{\text {ot }}$, but otherwise the protocol is fairly straight-forward; see Appendix B.

Malicious Setting. For the malicious setting we use ideas from the work of Nielsen et al. [NNOB12. The key notion is that of an (obliviously) authenticated bit, or aBit for short. Namely, for a bit $b$ authenticated towards $P_{i}, P_{i}$ holds both the bit $b$ and a mask $M_{b}$, with $P_{j}$ holding the authentication key $K_{b}$ and a global key $\Delta_{j}$ such that $M_{b}=K_{b} \oplus b \Delta_{j}$. Authenticated bits can be efficiently constructed in the random oracle model using OT extension [IKNP03, NNOB12. Besides the one-time cost of using OT extension, Nielsen et al. NNOB12 claim a protocol requiring only 8 calls to the underlying hash function $H$ per aBit $2^{2}$

In the following, we briefly describe how to instantiate our hybrid functionalities assuming sufficiently many aBits. We do not discuss $\mathcal{F}_{\mathbf{c f}}, \mathcal{F}_{\mathbf{o t}}$, and $\mathcal{F}_{\text {zkpok }}$ here because they can be instantiated without using aBits.

- $\mathcal{F}_{\text {const }}, \mathcal{F}_{\text {ss }}, \mathcal{F}_{\text {rand }}, \mathcal{F}_{\text {gate }}^{G}$. These hybrids can be directly instantiated using known protocols NNOB12, (we assume when using $\mathcal{F}_{\text {gate }}$ that only AND and XOR gates are used). We now describe the cost of these protocols in terms of the number of calls to the underlying hash function $H: \mathcal{F}_{\text {const }}$ requires no calls to $H ; \mathcal{F}_{\text {ss }}$ requires one aBit and thus 8 calls to $H ; \mathcal{F}_{\text {rand }}$ requires two aBits and thus 16 calls to $H ; \mathcal{F}_{\text {gate }}^{X O R}$ can be computed locally; and $\mathcal{F}_{\text {gate }}^{A N D}$ requires 584 calls to $H$.

- $\mathcal{F}_{\text {oshare }}$ The implementation of $\mathcal{F}_{\text {oshare }}$ requires calls to an appropriate underlying OT primitive. To ensure that the sharings are shuffled according to the authenticated bit, we introduce a primitive called receiver-authenticated string oblivious transfer, or in short, RaOT. As the name suggests, RaOT receives two input strings $s_{0}, s_{1} \in\{0,1\}^{*}$ from the sender $P_{j}$, and an aBit $\langle b\rangle^{(i)}$ from the receiver $P_{i}$, and outputs $s_{b}$ to the receiver (i.e., the aBit commits the receiver to his selection).

A naïve implementation of RaOT can be obtained by using the authenticated OT primitive from NNOB for each bit of the strings $s_{0}$ and $s_{1}$. However, this is a clear overkill as we only need the bit of the receiver to be authenticated. We have a more efficient implementation of $\mathrm{RaOT}$ which requires one aBit plus four additional calls to $H$, see Appendix C. $\mathcal{F}_{\text {oshare }}$ can be instantiated using two calls to RaOT and a single aBit; again, see Appendix C. Thus, $\mathcal{F}_{\text {oshare }}$ requires a total of 32 calls to $H$.

\section{Acknowledgments}

Work of Seung Geol Choi supported in part by the Office of Naval Research under Grant Number N0001414WX20588. Work of Jonathan Katz supported in part by NSF awards \#0964541 and \#1111599. Work of Alex J. Malozemoff supported by a National Defense Science and Engineering Graduate (NDSEG) Fellowship, 32 CFG 168a, awarded by DoD, Air Force Office of Scientific Research. Work of Vassilis Zikas supported in part by NSF awards \#09165174, \#1065276, \#1118126, and \#1136174, US-Israel BSF grant \#2008411, OKAWA Foundation Research Award, IBM Faculty Research Award, Xerox Faculty Research Award, B. John Garrick Foundation Award, Teradata Research Award, Lockheed-Martin Corporation Research Award, the Defense Advanced Research Projects Agency through the U.S. Office of Naval Research under Contract N00014-11-1-0392, and

\footnotetext{
${ }^{2}$ The protocol presented by Nielsen et al. NNOB12] uses 59 calls to $H$.
} 
Swiss National Science Foundation (SNF) Ambizione grant PZ00P2_142549. The views expressed are those of the authors and do not reflect the official policy or position of the Department of Defense or the U.S. Government.

\section{References}

[AL07] Yonatan Aumann and Yehuda Lindell. Security against covert adversaries: Efficient protocols for realistic adversaries. In Salil P. Vadhan, editor, TCC 2007: 4th Theory of Cryptography Conference, volume 4392 of Lecture Notes in Computer Science, pages 137-156, Amsterdam, The Netherlands, February 21-24, 2007. Springer-Verlag. Full version available at https://eprint.iacr.org/2007/060.

$\left[\mathrm{BCD}^{+}\right.$09] Peter Bogetoft, Dan Lund Christensen, Ivan Damgård, Martin Geisler, Thomas Jakobsen, Mikkel Krøigaard, Janus Dam Nielsen, Jesper Buus Nielsen, Kurt Nielsen, Jakob Pagter, Michael I. Schwartzbach, and Tomas Toft. Secure multiparty computation goes live. In Roger Dingledine and Philippe Golle, editors, 13th Intl. Conference on Financial Cryptography and Data Security, volume 5628 of Lecture Notes in Computer Science, pages 325-343, Accra Beach, Barbados, February 23-26, 2009. Springer-Verlag.

[BDNP08] Assaf Ben-David, Noam Nisan, and Benny Pinkas. FairplayMP: a system for secure multi-party computation. In Peng Ning, Paul F. Syverson, and Somesh Jha, editors, 15th ACM Conference on Computer and Communications Security, pages 257-266, Alexandria, Virginia, USA, October 27-31, 2008. ACM Press.

[BDOZ11] Rikke Bendlin, Ivan Damgård, Claudio Orlandi, and Sarah Zakarias. Semi-homomorphic encryption and multiparty computation. In Kenneth G. Paterson, editor, Advances in Cryptology-Eurocrypt 2011, volume 6632 of Lecture Notes in Computer Science, pages 169-188, Tallinn, Estonia, May 15-19, 2011. Springer-Verlag. Full version available at https://eprint.iacr.org/2010/514.

[BHR12] Mihir Bellare, Viet Tung Hoang, and Phillip Rogaway. Foundations of garbled circuits. In Ting Yu, George Danezis, and Virgil D. Gligor, editors, 19th ACM Conference on Computer and Communications Security, pages 784-796, Raleigh, NC, USA, October 1618, 2012. ACM Press. Full version available at https://eprint.iacr.org/2012/265.

[BLW08] Dan Bogdanov, Sven Laur, and Jan Willemson. Sharemind: A framework for fast privacy-preserving computations. In Sushil Jajodia and Javier López, editors, 13th European Symposium on Research in Computer Security (ESORICS), volume 5283 of Lecture Notes in Computer Science, pages 192-206, Málaga, Spain, October 6-8, 2008. Springer-Verlag. Full version available at https://eprint.iacr.org/2008/289.

[BMR90] Donald Beaver, Silvio Micali, and Phillip Rogaway. The round complexity of secure protocols (extended abstract). In 22nd Annual ACM Symposium on Theory of Computing, pages 503-513, Baltimore, Maryland, USA, May 14-16, 1990. ACM Press.

[BSMD10] Martin Burkhart, Mario Strasser, Dilip Many, and Xenofontas Dimitropoulos. SEPIA: Privacy-preserving aggregation of multi-domain network events and statistics. In 
Ian Goldberg, editor, 19th USENIX Security Symposium, Washington, D.C., USA, August 11-13, 2010. USENIX Association.

[BTW12] Dan Bogdanov, Riivo Talviste, and Jan Willemson. Deploying secure multi-party computation for financial data analysis - (short paper). In Angelos D. Keromytis, editor, 16th Intl. Conference on Financial Cryptography and Data Security, volume 7397 of Lecture Notes in Computer Science, pages 57-64, Kralendijk, Bonaire, February 27 March 2, 2012. Springer-Verlag. Full version available at https://eprint.iacr.org/ $2011 / 662$.

[Can00] Ran Canetti. Security and composition of multiparty cryptographic protocols. Journal of Cryptology, 13(1):143-202, 2000. Full version available at https://eprint.iacr. org/1998/018.

[Can01] Ran Canetti. Universally composable security: A new paradigm for cryptographic protocols. In 42nd Annual Symposium on Foundations of Computer Science, pages 136-145, Las Vegas, Nevada, USA, October 14-17, 2001. IEEE Computer Society Press. Full version available at https://eprint.iacr.org/2000/067.

$\left[\mathrm{CHK}^{+} 12\right]$ Seung Geol Choi, Kyung-Wook Hwang, Jonathan Katz, Tal Malkin, and Dan Rubenstein. Secure multi-party computation of Boolean circuits with applications to privacy in online marketplaces. In Orr Dunkelman, editor, Topics in Cryptology-CT-RSA 2012, volume 7178 of Lecture Notes in Computer Science, pages 416-432, San Francisco, CA, USA, February 27 - March 2, 2012. Springer-Verlag. Full version available at https://eprint.iacr.org/2011/257.

[DGKN09] Ivan Damgård, Martin Geisler, Mikkel Krøigaard, and Jesper Buus Nielsen. Asynchronous multiparty computation: Theory and implementation. In Stanislaw Jarecki and Gene Tsudik, editors, 12th International Conference on Theory and Practice of Public Key Cryptography, volume 5443 of Lecture Notes in Computer Science, pages 160-179, Irvine, CA, USA, March 18-20, 2009. Springer-Verlag. Full version available at https://eprint.iacr.org/2008/415.

[DI05] Ivan Damgård and Yuval Ishai. Constant-round multiparty computation using a black-box pseudorandom generator. In Victor Shoup, editor, Advances in CryptologyCrypto 2005, volume 3621 of Lecture Notes in Computer Science, pages 378-394, Santa Barbara, CA, USA, August 14-18, 2005. Springer-Verlag. Full version available at https://eprint.iacr.org/2005/262.

[DKL $\left.{ }^{+} 12\right]$ Ivan Damgård, Marcel Keller, Enrique Larraia, Christian Miles, and Nigel P. Smart. Implementing AES via an actively/covertly secure dishonest-majority MPC protocol. In Ivan Visconti and Roberto De Prisco, editors, 8th International Conference on Security in Communication Networks, volume 7485 of Lecture Notes in Computer Science, pages 241-263, Amalfi, Italy, September 5-7, 2012. Springer-Verlag. Full version available at https://eprint.iacr.org/2012/262.

[DKL $\left.{ }^{+} 13\right]$ Ivan Damgård, Marcel Keller, Enrique Larraia, Valerio Pastro, Peter Scholl, and Nigel P. Smart. Practical covertly secure MPC for dishonest majority - or: Breaking the SPDZ 
limits. In Jason Crampton, Sushil Jajodia, and Keith Mayes, editors, 18th European Symposium on Research in Computer Security (ESORICS), volume 8134 of Lecture Notes in Computer Science, pages 1-18, Egham, UK, September 9-13, 2013. Springer-Verlag. Full version available at https://eprint.iacr.org/2012/642.

[DPSZ12] Ivan Damgård, Valerio Pastro, Nigel P. Smart, and Sarah Zakarias. Multiparty computation from somewhat homomorphic encryption. In Reihaneh Safavi-Naini and Ran Canetti, editors, Advances in Cryptology-Crypto 2012, volume 7417 of Lecture Notes in Computer Science, pages 643-662, Santa Barbara, CA, USA, August 19-23, 2012. Springer-Verlag. Full version available at https://eprint.iacr.org/2011/535.

[DZ13] Ivan Damgård and Sarah Zakarias. Constant-overhead secure computation of boolean circuits using preprocessing. In Amit Sahai, editor, 10th Theory of Cryptography Conference, volume 7785 of Lecture Notes in Computer Science, pages 621-641, Tokyo, Japan, March 3-6, 2013. Springer-Verlag. Full version available at https://eprint. iacr.org/2012/512.

[GMS08] Vipul Goyal, Payman Mohassel, and Adam Smith. Efficient two party and multi party computation against covert adversaries. In Nigel P. Smart, editor, Advances in Cryptology-Eurocrypt 2008, volume 4965 of Lecture Notes in Computer Science, pages 289-306, Istanbul, Turkey, April 13-17, 2008. Springer-Verlag.

[GMW87] Oded Goldreich, Silvio Micali, and Avi Wigderson. How to play any mental game or A completeness theorem for protocols with honest majority. In Alfred Aho, editor, 19th Annual ACM Symposium on Theory of Computing, pages 218-229, New York City,, New York, USA, May 25-27, 1987. ACM Press.

[Gol09] Oded Goldreich. Foundations of Cryptography: Volume 2, Basic Applications, volume 2. Cambridge University Press, 2009.

[HEK12] Yan Huang, David Evans, and Jonathan Katz. Private set intersection: Are garbled circuits better than custom protocols? In ISOC Network and Distributed System Security Symposium - NDSS 2012, San Diego, California, USA, February 5-8, 2012. The Internet Society.

[HEKM11] Yan Huang, David Evans, Jonathan Katz, and Lior Malka. Faster secure two-party computation using garbled circuits. In David Wagner, editor, 20th USENIX Security Symposium, San Francisco, California, USA, August 8-12, 2011. USENIX Association.

[HKE13] Yan Huang, Jonathan Katz, and David Evans. Efficient secure two-party computation using symmetric cut-and-choose. In Ran Canetti and Juan A. Garay, editors, Advances in Cryptology - Crypto 2013, Part II, volume 8043 of Lecture Notes in Computer Science, pages 18-35, Santa Barbara, CA, USA, August 18-22, 2013. Springer-Verlag. Full version available at https://eprint.iacr.org/2013/081.

$\left[\mathrm{HKS}^{+} 10\right]$ Wilko Henecka, Stefan Kögl, Ahmad-Reza Sadeghi, Thomas Schneider, and Immo Wehrenberg. TASTY: tool for automating secure two-party computations. In Ehab Al-Shaer, Angelos D. Keromytis, and Vitaly Shmatikov, editors, 17th ACM Conference on Computer and Communications Security, pages 451-462, Chicago, Illinois, USA, 
October 4-8, 2010. ACM Press. Full version available at https://eprint.iacr.org/ $2010 / 365$

[IKNP03] Yuval Ishai, Joe Kilian, Kobbi Nissim, and Erez Petrank. Extending oblivious transfers efficiently. In Dan Boneh, editor, Advances in Cryptology-Crypto 2003, volume 2729 of Lecture Notes in Computer Science, pages 145-161, Santa Barbara, CA, USA, August $17-$ 21, 2003. Springer-Verlag.

[IPS08] Yuval Ishai, Manoj Prabhakaran, and Amit Sahai. Founding cryptography on oblivious transfer - efficiently. In David Wagner, editor, Advances in Cryptology-Crypto 2008, volume 5157 of Lecture Notes in Computer Science, pages 572-591, Santa Barbara, CA, USA, August 17-21, 2008. Springer-Verlag.

[KS08] Vladimir Kolesnikov and Thomas Schneider. Improved garbled circuit: Free XOR gates and applications. In Luca Aceto, Ivan Damgård, Leslie Ann Goldberg, Magnús M. Halldórsson, Anna Ingólfsdóttir, and Igor Walukiewicz, editors, ICALP 2008: 35th International Colloquium on Automata, Languages and Programming, Part II, volume 5126 of Lecture Notes in Computer Science, pages 486-498, Reykjavik, Iceland, July 7-11, 2008. Springer-Verlag.

[KsS12] Benjamin Kreuter, abhi shelat, and Chih-Hao Shen. Towards billion-gate secure computation with malicious adversaries. In Tadayoshi Kohno, editor, 21st USENIX Security Symposium, Bellevue, Washington, USA, August 8-10, 2012. USENIX Association. Full version available at https://eprint.iacr.org/2012/179.

[KSS13] Marcel Keller, Peter Scholl, and Nigel P. Smart. An architecture for practical actively secure MPC with dishonest majority. In Ahmad-Reza Sadeghi, Virgil D. Gligor, and Moti Yung, editors, 20th ACM Conference on Computer and Communications Security, pages 549-560, Berlin, Germany, November 4-8, 2013. ACM Press. Full version available at https://eprint.iacr.org/2013/143.

[Lin13] Yehuda Lindell. Fast cut-and-choose based protocols for malicious and covert adversaries. In Ran Canetti and Juan A. Garay, editors, Advances in Cryptology - Crypto 2013, Part II, volume 8043 of Lecture Notes in Computer Science, pages 1-17, Santa Barbara, CA, USA, August 18-22, 2013. Springer-Verlag.

[LOP11] Yehuda Lindell, Eli Oxman, and Benny Pinkas. The IPS compiler: Optimizations, variants and concrete efficiency. In Phillip Rogaway, editor, Advances in CryptologyCrypto 2011, volume 6841 of Lecture Notes in Computer Science, pages 259-276, Santa Barbara, CA, USA, August 14-18, 2011. Springer-Verlag. Full version available at https://eprint.iacr.org/2011/435.

[LP07] Yehuda Lindell and Benny Pinkas. An efficient protocol for secure two-party computation in the presence of malicious adversaries. In Moni Naor, editor, Advances in CryptologyEurocrypt 2007, volume 4515 of Lecture Notes in Computer Science, pages 52-78, Barcelona, Spain, May 20-24, 2007. Springer-Verlag.

[LP09] Yehuda Lindell and Benny Pinkas. A proof of security of Yao's protocol for two-party computation. Journal of Cryptology, 22(2):161-188, April 2009. 
[LP11] Yehuda Lindell and Benny Pinkas. Secure two-party computation via cut-and-choose oblivious transfer. In Yuval Ishai, editor, 8th Theory of Cryptography Conference, volume 6597 of Lecture Notes in Computer Science, pages 329-346, Providence, RI, USA, March 28-30, 2011. Springer-Verlag. Full version available at https://eprint. iacr.org/2010/284.

[LPS08] Yehuda Lindell, Benny Pinkas, and Nigel P. Smart. Implementing two-party computation efficiently with security against malicious adversaries. In Rafail Ostrovsky, Roberto De Prisco, and Ivan Visconti, editors, 6th International Conference on Security in Communication Networks, volume 5229 of Lecture Notes in Computer Science, pages 2-20, Amalfi, Italy, September 10-12, 2008. Springer-Verlag.

[MF06] Payman Mohassel and Matthew Franklin. Efficiency tradeoffs for malicious two-party computation. In Moti Yung, Yevgeniy Dodis, Aggelos Kiayias, and Tal Malkin, editors, 9th International Conference on Theory and Practice of Public Key Cryptography, volume 3958 of Lecture Notes in Computer Science, pages 458-473, New York, NY, USA, April 24-26, 2006. Springer-Verlag.

[MR13] Payman Mohassel and Ben Riva. Garbled circuits checking garbled circuits: More efficient and secure two-party computation. In Ran Canetti and Juan A. Garay, editors, Advances in Cryptology - Crypto 2013, Part II, volume 8043 of Lecture Notes in Computer Science, pages 36-53, Santa Barbara, CA, USA, August 18-22, 2013. Springer-Verlag. Full version available at https://eprint.iacr.org/2013/051.

[NNOB12] Jesper Buus Nielsen, Peter Sebastian Nordholt, Claudio Orlandi, and Sai Sheshank Burra. A new approach to practical active-secure two-party computation. In Reihaneh Safavi-Naini and Ran Canetti, editors, Advances in Cryptology-Crypto 2012, volume 7417 of Lecture Notes in Computer Science, pages 681-700, Santa Barbara, CA, USA, August 19-23, 2012. Springer-Verlag. Full version available at https://eprint.iacr. org/2011/091.

[NO09] Jesper Buus Nielsen and Claudio Orlandi. LEGO for two-party secure computation. In Omer Reingold, editor, 6th Theory of Cryptography Conference, volume 5444 of Lecture Notes in Computer Science, pages 368-386. Springer-Verlag, March 15-17, 2009. Full version available at https://eprint.iacr.org/2008/427.

[PSSW09] Benny Pinkas, Thomas Schneider, Nigel P. Smart, and Stephen C. Williams. Secure two-party computation is practical. In Mitsuru Matsui, editor, Advances in Cryptology - ASIACRYPT 2009, volume 5912 of Lecture Notes in Computer Science, pages 250267, Tokyo, Japan, December 6-10, 2009. Springer-Verlag. Full version available at https://eprint.iacr.org/2009/314.

[PVW08] Chris Peikert, Vinod Vaikuntanathan, and Brent Waters. A framework for efficient and composable oblivious transfer. In David Wagner, editor, Advances in CryptologyCrypto 2008, volume 5157 of Lecture Notes in Computer Science, pages 554-571, Santa Barbara, CA, USA, August 17-21, 2008. Springer-Verlag. Full version available at https://eprint.iacr.org/2007/348. 
[sS11] abhi shelat and Chih-Hao Shen. Two-output secure computation with malicious adversaries. In Kenneth G. Paterson, editor, Advances in Cryptology-Eurocrypt 2011, volume 6632 of Lecture Notes in Computer Science, pages 386-405, Tallinn, Estonia, May 15-19, 2011. Springer-Verlag. Full version available at https://eprint.iacr.org/2011/533.

[sS13] abhi shelat and Chih-Hao Shen. Fast two-party secure computation with minimal assumptions. In Ahmad-Reza Sadeghi, Virgil D. Gligor, and Moti Yung, editors, 20th ACM Conference on Computer and Communications Security, pages 523-534, Berlin, Germany, November 4-8, 2013. ACM Press. Full version available at https: //eprint.iacr.org/2013/196.

[SZ13] Thomas Schneider and Michael Zohner. GMW vs. Yao? efficient secure two-party computation with low depth circuits. In Ahmad-Reza Sadeghi, editor, 17th Intl. Conference on Financial Cryptography and Data Security, volume 7859 of Lecture Notes in Computer Science, pages 275-292, Okinawa, Japan, April 1-5, 2013. Springer-Verlag.

[Woo07] David P. Woodruff. Revisiting the efficiency of malicious two-party computation. In Moni Naor, editor, Advances in Cryptology-Eurocrypt 2007, volume 4515 of Lecture Notes in Computer Science, pages 79-96, Barcelona, Spain, May 20-24, 2007. Springer-Verlag. Full version available at https://eprint.iacr.org/2006/397.

[Yao86] Andrew Chi-Chih Yao. How to generate and exchange secrets (extended abstract). In 27th Annual Symposium on Foundations of Computer Science, pages 162-167, Toronto, Ontario, Canada, October 27-29, 1986. IEEE Computer Society Press. 


\section{Appendices}

\section{A Hybrid Functionalities}

Secret Sharing of a Constant Bit. The functionality $\mathcal{F}_{\text {const }}^{b}$ is parameterized by a bit $b$, and outputs a sharing (authenticated, in the malicious setting) of that bit.

Functionality $\mathcal{F}_{\text {const }}^{b} \rightarrow\langle b\rangle$

Output: The functionality does the following:

1. $\quad$ Choose bit $r \leftarrow \$\{0,1\}$.

2. $\quad$ (Semi-honest setting) Output $r$ to $P_{i}$ and $r \oplus b$ to $P_{j}$.

3. (Malicious setting) Construct authenticated bits $r_{i}=\langle r\rangle^{(i)}$ and $r_{j}=\langle r \oplus b\rangle^{(j)}$, and output $r_{i}$ to party $P_{i}$ and $r_{j}$ to party $P_{j}$.

In the semi-honest setting, this functionality can be instantiated by having $P_{i}$ generate a random bit $r$ and sending $r$ to $P_{j}$, who computes $r \oplus b$. In the malicious setting we can instantiate this using the protocol described by Nielsen et al. [NNOB12, Figure 3].

Bit Secret Sharing. The functionality $\mathcal{F}_{\text {ss }}$ in the semi-honest setting is the standard secret sharing functionality. In the malicious setting, the functionality creates an authenticated sharing of the input bit.

Functionality $\mathcal{F}_{\mathbf{s s}}^{i}(b) \rightarrow\langle b\rangle$

Input: Party $P_{i}$ inputs a bit $b$.

Output: The functionality does the following:

1. Select $r \leftarrow \$\{0,1\}$ uniformly at random.

2. (Semi-honest setting) Output $r$ to party $P_{j}$, and $b \oplus r$ to party $P_{i}$.

3. (Malicious setting) Construct authenticated bits $b_{j}=\langle r\rangle^{(j)}$ and $b_{i}=\langle b \oplus r\rangle^{(i)}$, and output $b_{j}$ to party $P_{j}$ and $b_{i}$ to party $P_{i}$.

Implementing the functionality in the semi-honest setting is trivial. In the malicious setting we can use the Input protocol described by Nielsen et al. [NNOB12, Figure 6].

One-Out-of-Two Oblivious Secret Sharing. The functionality $\mathcal{F}_{\text {oshare }}$ is used to share the sub-keys of the garbled table in an oblivious fashion while preserving consistency with respect to the circuit such that the circuit evaluation succeeds given the correct input sub-keys. More precisely, $\mathcal{F}_{\text {oshare }}$ interacts with two parties, called the sender $P_{j}$ and the receiver $P_{i}$; it expects two inputs, $m_{0}$ and $m_{1}$, from the sender along with a two-out-of-two sharing $\langle b\rangle$ of a selection bit $b$ between the sender and receiver, and outputs a random two-out-of-two sharing $\left[m_{b}\right]$ of $m_{b}$. In the malicious setting, $\langle b\rangle$ is an authenticated bit share. The functionality does not leak any information about $b$ to the parties. Furthermore, when the sender is honest, it leaks no information on its inputs to the receiver. However, to ensure simulatability we allow a corrupted sender to choose his output share, $y^{(j)}$, at will. 
$\underline{\text { Functionality } \mathcal{F}_{\text {oshare }}^{i, j}\left(\langle b\rangle^{(i)},\left(\langle b\rangle^{(j)}, m_{0}, m_{1}, y^{(j)}\right)\right) \rightarrow\left[m_{b}\right]}$

Input: Party $P_{i}$ inputs share $b_{i}=\langle b\rangle^{(i)}$. Party $P_{j}$ inputs share $b_{j}=\langle b\rangle^{(j)}$ and vector $\left(m_{0}, m_{1}\right) \in$ $\{0,1\}^{k} \times\{0,1\}^{k}$. In the malicious setting, $b_{i}$ and $b_{j}$ are authenticated. Additionally, party $P_{j}$ input a value $y^{(j)} \in\{0,1\}^{k} \cup\{\perp\}$; if $P_{j}$ is honest he sets $y^{(j)}=\perp$, otherwise $y^{(j)}$ can be arbitrary.

Output: The functionality does the following:

1. (Malicious setting) If either $\langle b\rangle^{(i)}$ or $\langle b\rangle^{(j)}$ is not a correctly authenticated bit then abort.

2. Compute $b=b_{i} \oplus b_{j}$

3. If $y^{(j)}=\perp$, then choose $y^{(j)} \leftarrow \$\{0,1\}^{k}$.

4. $\quad$ Output $m_{b} \oplus y^{(j)}$ to $P_{i}$ and $y^{(j)}$ to $P_{j}$.

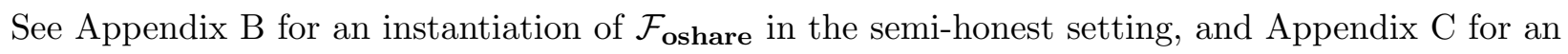
instantiation in the malicious setting.

Oblivious Secret Sharing. The oblivious secret sharing functionality $\mathcal{F}_{\text {rand }}$ takes no inputs, and outputs a sharing of a random bit. In the malicious setting, this output sharing is authenticated.

$\underline{\text { Functionality } \mathcal{F}_{\text {rand }} \rightarrow\langle r\rangle}$

Output: The functionality does the following:

1. Choose bits $r, r^{\prime} \leftarrow \$\{0,1\}$.

2. (Semi-honest setting) Output $r^{\prime}$ to $P_{i}$ and $r \oplus r^{\prime}$ to $P_{j}$.

3. (Malicious setting) Construct authenticated bits $r_{i}=\left\langle r^{\prime}\right\rangle^{(i)}$ and $r_{j}=\left\langle r \oplus r^{\prime}\right\rangle^{(j)}$, and output $r_{i}$ to party $P_{i}$ and $r_{j}$ to party $P_{j}$.

In the semi-honest setting, this can be easily instantiated by each party choosing a random bit $r_{i}$ and letting $r=r_{i} \oplus r_{j}$. In the malicious setting we need to construct authenticated shares and thus need additional machinery: we can utilize the Rand protocol by Nielsen et al. [NNOB12, Figure 6].

Oblivious Gate Evaluation. The functionality $\mathcal{F}_{\text {gate }}^{G}$ takes as inputs shares of bits $a$ and $b$, and outputs a share of $G(a, b)$ for some binary gate $G$. In the malicious setting, both input and output shares are authenticated.

Functionality $\mathcal{F}_{\text {gate }}^{G}(\langle a\rangle,\langle b\rangle) \rightarrow\langle G(a, b)\rangle$

Input: The parties input bit shares $\langle a\rangle$ and $\langle b\rangle$. In the malicious setting, these shares are authenticated.

Auxiliary Input: The description of a binary gate $G$.

Output: The functionality does the following:

1. (Malicious setting) If any of the shares are not correctly authenticated then abort.

2. Compute $a$ and $b$ from the shares.

3. Compute $c=G(a, b)$ and output a sharing (authenticated in the malicious setting) of $c$.

As our circuits require only AND and XOR gates, we only consider those choices for $G$ here. In the semi-honest setting, we can compute XOR gates locally and AND gates using one-out-of-four OT, as is done in the GMW protocol [GMW87. In the malicious setting we can efficiently instantiate the functionality for XOR and AND gates using the XOR and AND protocols by Nielsen et al. [NNOB12, Figure 6].

Oblivious Transfer. The functionality $\mathcal{F}_{\text {ot }}$ implements standard oblivious transfer. 
Functionality $\mathcal{F}_{\text {ot }}^{i, j}\left(b,\left(m_{0}, m_{1}\right)\right) \rightarrow m_{b}$

Input: Party $P_{i}$ inputs a choice bit $b$. Party $P_{j}$ inputs two messages $m_{0}$ and $m_{1}$.

Output: The functionality outputs $m_{b}$ to $P_{i}$, and $\perp$ to $P_{j}$.

This can be implemented efficiently in both the semi-honest and malicious setting using known existing protocols [PVW08].

\section{B Hybrid Implementations for the Semi-Honest Protocol}

One-Out-of-Two Oblivious Secret Sharing. The following protocol implements the $\mathcal{F}_{\text {oshare }}$ functionality:

$$
\text { Protocol } \Pi_{\text {oshare }}^{i, j}\left(\langle b\rangle^{(i)},\left(\langle b\rangle^{(j)}, m_{0}, m_{1}, \perp\right)\right)
$$

Input: Party $P_{i}$ inputs his share $b_{i}=\langle b\rangle^{(i)}$ of $\langle b\rangle$. Party $P_{j}$ inputs his share $b_{j}=\langle b\rangle^{(j)}$ of $\langle b\rangle$ along with two strings $m_{0}, m_{1} \in\{0,1\}^{k}$.

1. $P_{j}$ chooses $r \leftarrow \$\{0,1\}^{k}$ uniformly at random.

2. Execute $\mathcal{F}_{\text {ot }}$ with $P_{j}$ as the sender having inputs $\left(s_{0}, s_{1}\right)=\left(m_{0} \oplus r, m_{1} \oplus r\right)$, and $P_{i}$ as the receiver having input $b^{\prime}=1 \oplus b_{i}$; denote $P_{i}$ 's output as $y_{i}$.

Outputs: $P_{j}$ outputs $y_{j}=\left(\left(m_{0} \oplus m_{1}\right) \odot\left(1 \oplus b_{j}\right)\right) \oplus r$ and $P_{i}$ outputs $y_{i}$.

Lemma 1. The protocol $\Pi_{\text {oshare }}^{i, j}$ securely implements the functionality $\mathcal{F}_{\text {oshare }}^{i, j}$ in the presence of a semi-honest adversary in the $\mathcal{F}_{\text {ot }}$-hybrid world.

Proof. First, we show correctness; namely, we argue that the output of the protocol is a two-out-oftwo sharing of $m_{b}$, that is, $y_{i} \oplus y_{j}=m_{b}$. Indeed,

$$
\begin{aligned}
y_{i} \oplus y_{j} & =m_{1 \oplus b_{i}} \oplus r \oplus\left(\left(m_{0} \oplus m_{1}\right) \odot\left(1 \oplus b_{j}\right)\right) \oplus r \\
& =m_{1 \oplus b_{i}} \oplus\left(\left(m_{0} \oplus m_{1}\right) \odot\left(1 \oplus b_{j}\right)\right) .
\end{aligned}
$$

Note that if $b_{j}=0$, we have

$$
y_{i} \oplus y_{j}=m_{1 \oplus b_{i}} \oplus m_{0} \oplus m_{1}=m_{b} .
$$

Likewise, if $b_{j}=1$, we have

$$
y_{i} \oplus y_{j}=m_{1 \oplus b_{i}}=m_{b} .
$$

To prove that the protocol is simulatable, observe that (1) $P_{j}$ receives no information in the protocol (which follows from the privacy of OT) and (2) $P_{i}$ only sees $y_{i}$, where the value $m_{1 \oplus b_{i}}$ is perfectly blinded by $r$. Hence, similarly to the ideal evaluation of $\mathcal{F}_{\text {oshare }}^{i, j}$, the values seen by the parties give them no information. More formally, we consider the following cases:

$P_{i}$ is corrupted: The simulator, emulating the execution of $\mathcal{F}_{\text {ot }}$ towards $P_{i}$, waits for $\mathcal{A}$ to input

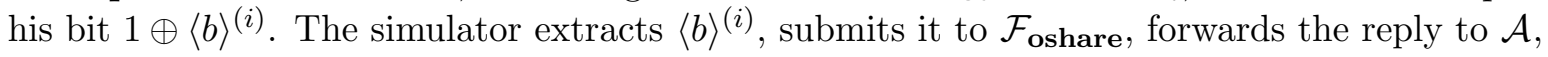
and halts with $\mathcal{A}$ 's output. 
$P_{j}$ is corrupted: The simulator receives the messages $\left(m_{0} \oplus r, m_{1} \oplus r\right)$ from $\mathcal{A}$ and extracts $r$, which is possible due to the semi-honest setting. The simulator then submits $\left(\langle b\rangle^{(j)}, m_{0}, m_{1}, y^{(j)}\right)$ to $\mathcal{F}_{\text {oshare }}$, where $y^{(j)}=\left(m_{0} \oplus m_{1}\right) \odot\left(1 \oplus\langle b\rangle^{(j)}\right) \oplus r$, and halts with $\mathcal{A}$ 's output.

Noting that each of these simulators perfectly simulates the adversary in the $\mathcal{F}_{\text {ot }}$-hybrid world, the protocol is secure.

\section{Hybrid Implementations for the Malicious Protocol}

In the malicious setting we utilize ideas from the protocol by Nielsen et al. [NNOB12. In particular, each party $P_{i}$ holds a global key $\Delta_{i}$, which they use to construct authenticated bit shares. For a bit $b$ authenticated towards $P_{i}, P_{i}$ holds both the bit $b$ and a MAC $M_{b}$, with $P_{j}$ holding the authentication key $K_{b}$, with the condition that $M_{b}=K_{b} \oplus b \Delta_{j}$. To ease notation, in this section we let $\langle b\rangle^{(i)}=\left(b, M_{b}, K_{b}\right)$.

Authenticated Bit. We repeat here the $\mathcal{F}_{\text {aBit }}$ functionality [NNOB12, Figure 5].

$\underline{\text { Functionality } \mathcal{F}_{\text {aBit }} \rightarrow\langle r\rangle^{(i)}}$

Auxiliary Input: Party $P_{j}$ inputs his global key $\Delta_{j} \leftarrow\{0,1\}^{k}$.

Output:

1. (If $P_{i}$ is malicious) Set $\langle b\rangle^{(i)}=(b, M, M \oplus b \Delta)$.

2. (If $P_{j}$ is malicious) Let $b \leftarrow \$\{0,1\}$ and set $\langle b\rangle^{(i)}=\left(b, K \oplus b \Delta_{j}, K\right)$.

3. (If both are honest) Let $b \leftarrow \$\{0,1\}$ and $K \leftarrow \$\{0,1\}^{k}$, and set $\langle b\rangle^{(i)}=\left(b, K \oplus b \Delta_{j}, K\right)$.

4. $\quad$ Output $\left(b, K \oplus b \Delta_{j}\right)$ to $P_{i}$ and $\left(K, \Delta_{j}\right)$ to $P_{j}$.

The implementation of $\mathcal{F}_{\text {aBit }}$ is detailed in the work of Nielsen et al. [NNOB12, Section 4] and not repeated here. Note that the parties can utilize $\mathcal{F}_{\text {aBit }}$ to construct a constant bit by $P_{i}$ setting $M=0^{k}$ and $P_{j}$ setting $K=b \Delta_{j}$.

Receiver-Authenticated One-Out-of-Two Oblivious Transfer. We first define the functionality for receiver-authenticated oblivious transfer:

Functionality $\mathcal{F}_{\text {raot }}^{i, j}\left(\langle b\rangle^{(i)},\left(m_{0}, m_{1}\right)\right) \rightarrow m_{b}$

Input: Party $P_{i}$ inputs an authenticated choice bit $b$. Party $P_{j}$ inputs two messages $m_{0}$ and $m_{1}$.

Output: The functionality does the following:

1. If $P_{i}$ 's choice bit $b$ is not correctly authenticated then abort.

2. Output $m_{b}$ to $P_{i}$.

In order to efficiently implement $\mathcal{F}_{\text {raot }}$, we need the following functionality:

Functionality $\mathcal{F}_{\text {eq }}(a, b) \rightarrow\{0,1\}$

Input: Party $P_{i}$ inputs $a \in\{0,1\}^{k}$, and party $P_{j}$ inputs $b \in\{0,1\}^{k}$.

Output: The functionality outputs 1 if $a=b$, and 0 otherwise. 
This can be instantiated efficiently using two calls to a random oracle $H$ [NNOB12, pg. 7]. We can thus instantiate $\mathcal{F}_{\text {raot }}$ as follows:

$$
\text { Protocol } \Pi_{\text {raot }}\left(\langle b\rangle^{(i)},\left(m_{0}, m_{1}\right)\right)
$$

Let $\langle b\rangle^{(i)}=\left(b, M_{b}, K_{b}\right)$.

1. The parties compute $\langle r\rangle^{(i)}=\left(r, M_{r}, K_{r}\right) \leftarrow \mathcal{F}_{\text {aBit }}^{i}$.

2. $P_{i}$ computes $d=b \oplus r$ and sends $d$ to $P_{j}$.

3. $P_{i}$ sends $M_{b} \oplus M_{r}$ to $\mathcal{F}_{\text {eq }}$, and $P_{j}$ sends $\left(K_{b} \oplus K_{r}\right) \oplus d \Delta_{j}$ to $\mathcal{F}_{\text {eq }}$, to check the equality of the two values. If they are not equal, $P_{j}$ aborts the protocol.

4. The parties then compute $\langle d\rangle^{(i)} \leftarrow \mathcal{F}_{\text {const }}^{d}$.

5. Let $\langle w\rangle^{(i)}=\langle r\rangle^{(i)} \oplus\langle d\rangle^{(i)}$. $P_{j}$ sends $X_{0}=H\left(K_{w}\right) \oplus m_{0}$ and $X_{1}=H\left(K_{w} \oplus \Delta_{j}\right) \oplus m_{1}$ to $P_{i}$.

Output: $P_{i}$ outputs $X_{w} \oplus H\left(M_{w}\right)$ and $P_{j}$ outputs $\perp$.

Lemma. The protocol $\Pi_{\text {raot }}$ securely implements the functionality $\mathcal{F}_{\text {raot }}$ in the presence of a malicious adversary in the Random Oracle model.

Proof. Noting that $w=b$ in the protocol, correctness is immediate. To prove simulatability, we consider the following corruption cases:

$P_{i}$ is corrupted: The simulator $\mathcal{S}$ simulating an adversary $\mathcal{A}$ corrupting $P_{i}$ proceeds as follows. $\mathcal{S}$ forwards its input $\left(b, M_{b}\right)$ to $\mathcal{A}$ as input to the protocol. If $\mathcal{A}$ sends a message $v$ to $\mathcal{F}_{\text {eq }}$ such that $v \neq M_{b} \oplus M_{r}, \mathcal{S}$ aborts the protocol. Otherwise, $\mathcal{S}$ sends $\langle b\rangle^{(i)}$ to the trusted third party, receiving back $m_{b}$. $\mathcal{S}$ then generates two random bit-strings $X_{0}$ and $X_{1}$, and programs $H$ so that $H\left(M_{w}\right)=X_{b} \oplus m_{b}$. Finally, $\mathcal{S}$ sends $X_{0}$ and $X_{1}$ to $\mathcal{A}$.

$P_{j}$ is corrupted: The simulator $\mathcal{S}$ simulating an adversary $\mathcal{A}$ corrupting $P_{j}$ proceeds as follows. $\mathcal{S}$ forwards its input $\left(m_{0}, m_{1}, K_{b}\right)$ to $\mathcal{A}$ as input to the protocol. If $\mathcal{A}$ sends a message $v$ to $\mathcal{F}_{\text {eq }}$ such that $v \neq\left(K_{b} \oplus K_{r}\right) \oplus d \Delta_{j}, \mathcal{S}$ aborts the protocol. Next, $\mathcal{S}$ waits until $P_{j}$ sends $X_{0}$ and $X_{1}$ to $P_{i}$. It then uses its knowledge of $\langle r\rangle^{(i)}$ and $\langle d\rangle^{(i)}$ to extract $m_{0}$ and $m_{1}$, which it feeds to the trusted third party.

Noting that each of these simulators perfectly simulate the adversary in the $\left(\mathcal{F}_{\text {aBit }}, \mathcal{F}_{\text {const }}, \mathcal{F}_{\text {eq }}\right)$ hybrid world, the protocol is secure.

One-Out-of-Two Oblivious Secret Sharing. We can instantiate $\mathcal{F}_{\text {oshare }}$ in the malicious setting using a protocol similar to $\Pi_{\text {oshare }}$ with the following modifications:

1. We use receiver-authenticated OT in place of standard OT;

2. To ensure that the simulator can extract consistent inputs from a corrupted sender, we do two invocations of $\mathcal{F}_{\text {raot }}$;

3. In order to extract $P_{j}$ 's input in the case $P_{j}$ is corrupt, we need an addition authentication check requiring one invocation of $\mathcal{F}_{\text {aBit }}$. 


$$
\text { Protocol } \Pi_{\text {oshare-m }}^{i, j}\left(\langle b\rangle^{(i)},\left(\langle b\rangle^{(j)}, m_{0}, m_{1}, \perp\right)\right)
$$

Let $\langle b\rangle^{(i)}=\left(b_{i}, M_{b_{i}}, K_{b_{i}}\right)$ and $\langle b\rangle^{(j)}=\left(b_{j}, M_{b_{j}}, K_{b_{j}}\right)$.

1. $P_{j}$ chooses $r_{0}, r_{1} \leftarrow \$\{0,1\}^{k}$ uniformly at random.

2. Execute $\mathcal{F}_{\text {raot }}$ with $P_{j}$ as sender having inputs $\left(s_{0}, s_{1}\right)=\left(m_{0} \oplus r_{0}, m_{1} \oplus r_{1}\right)$, and $P_{i}$ as the receiver having input $\langle 1\rangle^{(i)} \oplus\langle b\rangle^{(i)} ; P_{i}$ denotes his output by $y_{i}$.

3. Execute $\mathcal{F}_{\text {raot }}$ with $P_{j}$ as sender having inputs $\left(s_{0}, s_{1}\right)=\left(r_{0}, r_{1}\right)$, and $P_{i}$ as the receiver having input $\langle b\rangle^{(i)}$; $P_{i}$ denotes his output by $r_{b_{i}}$.

4. Execute $\langle r\rangle^{(j)}=\left(r, M_{r}, K_{r}\right) \leftarrow \mathcal{F}_{\text {aBit }}^{j} . P_{j}$ sends $\left(d, M_{d}\right)$ to $P_{i}$, where $d=r \oplus b_{j}$ and $M_{d}=M_{r} \oplus M_{b_{j}}$, and $P_{i}$ checks if $\left(d, M_{d}, K_{d} \oplus K_{r}\right)$ is a valid authenticated bit, aborting if not.

Outputs: $P_{j}$ outputs $y^{(j)}=\left(\left(m_{0} \oplus m_{1}\right) \odot\left(1 \oplus b_{j}\right)\right) \oplus r_{0} \oplus r_{1}$ and $P_{i}$ outputs $y_{i} \oplus r_{b_{i}}$.

Lemma. The protocol $\Pi_{\text {oshare-m }}$ securely implements the functionality $\mathcal{F}_{\text {oshare }}$ in the presence of a malicious adversary in the $\left(\mathcal{F}_{\text {aBit }}, \mathcal{F}_{\text {raot }}\right)$-hybrid world.

Proof. We first demonstrate correctness. It suffices to show that $y_{i} \oplus y_{j}=m_{b}$. Indeed,

$$
\begin{aligned}
y_{i} \oplus y_{j} & =\left(m_{1 \oplus b_{i}} \oplus r_{1 \oplus b_{i}} \oplus r_{b_{i}}\right) \oplus\left(\left(m_{0} \oplus m_{1}\right) \odot\left(1 \oplus b_{j}\right)\right) \oplus r_{0} \oplus r_{1} \\
& =m_{1 \oplus b_{i}} \oplus\left(\left(m_{0} \oplus m_{1}\right) \odot\left(1 \oplus b_{j}\right)\right),
\end{aligned}
$$

and the derivation follows exactly as in the semi-honest case.

To prove that the protocol is simulatable, we consider the following corruption cases:

$P_{i}$ is corrupted: The simulator $\mathcal{S}$ waits for $\mathcal{A}$ to input his choice bit $\left\langle b^{\prime}\right\rangle^{(i)}$ to the first $\mathcal{F}_{\text {raot }}$ invocation. If $\left\langle b^{\prime}\right\rangle$ is not a valid authenticated bit, $\mathcal{S}$ aborts. Otherwise, $\mathcal{S}$ returns to $\mathcal{A}$ a

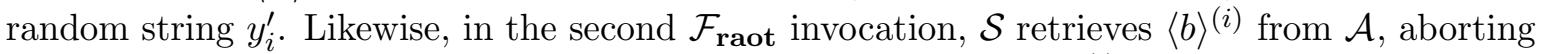
if the authentication check fails. $\mathcal{S}$ then invokes $\mathcal{F}_{\text {oshare }}$ with $\langle b\rangle^{(i)}$, receiving $P_{i}$ 's output $y_{i}$. $\mathcal{S}$ computes $r_{i}^{\prime}=y_{i} \oplus y_{i}^{\prime}$ and sends $r_{i}^{\prime}$ to $\mathcal{A}$ as the output of the second $\mathcal{F}_{\text {raot }}$. Finally, $\mathcal{S}$ acts as $P_{j}$ would in Step 4 , and halts with $\mathcal{A}$ 's output.

$P_{j}$ is corrupted: The simulator $\mathcal{S}$ emulates the two executions of $\mathcal{F}_{\text {raot }}$ towards the adversary $\mathcal{A}$ controlling $P_{j}$, from which $\mathcal{S}$ receives $\left(x_{0}, x_{1}\right)$ and $\left(r_{0}^{\prime}, r_{1}^{\prime}\right)$, respectively. In Step 4, $\mathcal{S}$ extracts $\left\langle b_{j}\right\rangle^{(j)}$. $\mathcal{S}$ then computes $\left(m_{0}^{\prime}, m_{1}^{\prime}\right)=\left(x_{0} \oplus r_{0}^{\prime}, x_{1} \oplus r_{1}^{\prime}\right)$ and submits the message $\left(\left\langle b_{j}\right\rangle^{(j)}, m_{0}^{\prime}, m_{1}^{\prime}, y^{(j)}\right)$ to $\mathcal{F}_{\text {oshare}}$, where $y^{(j)}=\left(\left(m_{0}^{\prime} \oplus m_{1}^{\prime}\right) \odot\left(1 \oplus b_{j}\right)\right) \oplus r_{0}^{\prime} \oplus r_{1}^{\prime}$, and halts with $\mathcal{A}$ 's output.

Noting that each of these simulators perfectly simulates the adversary in the $\left(\mathcal{F}_{\text {aBit }}, \mathcal{F}_{\text {raot }}\right)$-hybrid world, the protocol is secure.

\section{2PC Protocol Using Distributed Garbled Circuits}

\section{D.1 The Semi-Honest Setting}

We describe a secure two-party computation protocol $\Pi_{\mathbf{2 P C}}\left(P_{1}, P_{2}\right)$ which uses our two-party distributed garbling scheme $\Pi_{\mathbf{G C}}\left(P_{1}, P_{2}\right)$ as a sub-protocol to securely compute any polynomial-size circuit $C$ toward $P_{2}$; see Figure 3 for the detailed description. The parties first perform a distributed 


\section{Protocol $\Pi_{2 \mathrm{PC}}\left(P_{1}, P_{2}\right)$}

Auxiliary Inputs: Security parameter $k$, circuit $(\mathrm{n}, \mathrm{m}, \mathrm{q}, L, R, G) \leftarrow C$.

Inputs: For $w \in\left\{1, \ldots, \mathbf{n}_{1}\right\}, P_{1}$ has inputs $b_{w}$; for $w \in\left\{\mathbf{n}_{1}+1, \ldots, \mathbf{n}\right\}, P_{2}$ has inputs $b_{w}$.

1. The parties execute $\Pi_{\mathbf{G C}}\left(P_{1}, P_{2}\right)$.

2. For $w \in\left\{1, \ldots, \mathrm{n}_{1}\right\}$ : The parties execute $\left\langle b_{w}\right\rangle \leftarrow \mathcal{F}_{\mathbf{s s}}^{1}\left(b_{w}\right)$.

3. For $w \in\left\{\mathbf{n}_{1}+1, \ldots, \mathbf{n}_{2}\right\}$ : The parties execute $\left\langle b_{w}\right\rangle \leftarrow \mathcal{F}_{\mathbf{s s}}^{2}\left(b_{w}\right)$.

4. $\quad P_{1}$ sends $G C^{1}$ to $P_{2}$.

5. For $w \in\left\{1, \ldots, \mathrm{n}_{1}\right\}: P_{1}$ sends $\left(s_{w, b_{w} \oplus \lambda_{w}}^{1},\left\langle b_{w}\right\rangle^{(1)} \oplus\left\langle\lambda_{w}\right\rangle^{(1)}\right)$ to $P_{2}$ who reconstructs $b_{w} \oplus \lambda_{w}$ locally.

6. For $w \in\left\{\mathbf{n}_{1}+1, \ldots, \mathbf{n}\right\}: P_{2}$ sends $\left\langle b_{w}\right\rangle^{(2)} \oplus\left\langle\lambda_{w}\right\rangle^{(2)}$ to $P_{1}$, who reconstructs $b_{w} \oplus \lambda_{w}$ locally. $P_{1}$ then sends $s_{w, b_{w} \oplus \lambda_{w}}^{1}$ to $P_{2}$.

7. $\quad P_{2}$ evaluates the garbled circuit using the keys $\left(s_{w, b_{w} \oplus \lambda_{w}}^{1}, s_{w, b_{w} \oplus \lambda_{w}}^{2}\right)$ and selector bits $b_{w} \oplus \lambda_{w}$, for $w \in\{1, \ldots, \mathbf{n}\}$.

Figure 3: Two-party secure function evaluation.

garbling of the circuit and secret-sharing of their inputs. The parties then exchange input keys as follows:

- For each of $P_{1}$ 's input wires, $P_{1}$ sends to $P_{2}$ the appropriate sub-key $s_{w, b_{w} \oplus \lambda_{w}}^{1}$, as well as his share $\left\langle b_{w}\right\rangle^{(1)} \oplus\left\langle\lambda_{w}\right\rangle^{(1)} ; P_{2}$ reconstructs $b_{w} \oplus \lambda_{w}$ and selects sub-key $s_{w, b_{w} \oplus \lambda_{w}}^{2}$ to use for decryption $\}^{3}$

- For each of $P_{2}$ 's input wires, we do a similar protocol as for $P_{1}$ 's input wires. Here, $P_{2}$ sends $\left\langle b_{w}\right\rangle^{(2)} \oplus\left\langle\lambda_{w}\right\rangle^{(2)}$ to $P_{1}$, who reconstructs $b_{w} \oplus \lambda_{w} ; P_{1}$ then sends sub-key $s_{w, b_{w} \oplus \lambda_{w}}^{1}$ to $P_{2}{ }^{4}$

Given the "shares" of the garbled circuit and the input keys, $P_{2}$ can evaluate the garbled circuit to retrieve the output.

Theorem 3. The protocol $\Pi_{\mathbf{2} \mathbf{P C}}\left(P_{1}, P_{2}\right)$ securely evaluates the circuit $C$ in the presence of a static, semi-honest adversary in the $\left(\mathcal{F}_{\text {const }}, \mathcal{F}_{\text {gate }}, \mathcal{F}_{\text {oshare }}, \mathcal{F}_{\text {ot }}, \mathcal{F}_{\text {rand }}, \mathcal{F}_{\text {ss }}\right)$-hybrid world.

Proof. Correctness of the construction can be easily verified by inspection of the protocol. In the remainder of the proof we describe (black-box straight-line) simulators $\mathcal{S}_{1}$ and $\mathcal{S}_{2}$ which simulate an adversary corrupting $P_{1}$ and $P_{2}$, respectively.

The simulator $\mathcal{S}_{1}$ : We simulate adversary $P_{1}^{*}$ corrupting $P_{1}$ as follows. $\mathcal{S}_{1}$ plays as $P_{2}$ and follows the protocol description using $0^{\mathrm{n}-\mathrm{n}_{1}}$ as input. The messages which $P_{1}^{*}$ sees during the simulation are all shares of random values and input values. Therefore, due to the property of the secret sharing, the simulation is perfect.

The simulator $\mathcal{S}_{2}$ : Simulating adversary $P_{2}^{*}$ corrupting $P_{2}$ is less trivial. In addition to running $\Pi_{\mathbf{G C}}, \mathcal{S}_{2}$ must also simulate sending $P_{1}$ 's share of the garbled circuit along with the correct keys and mask bits that $P_{1}$ sends to $P_{2}^{*}$ after the execution of $\Pi_{\mathrm{GC}}$. However, $\mathcal{S}_{2}$ does not know the inputs of $P_{1}$. Hence, $\mathcal{S}_{2}$ has to come up with a sharing of a "fake" circuit which is indistinguishable from the original circuit. We handle this as follows. $\mathcal{S}_{2}$ uses 0 for each of $P_{1}$ 's input bits, which would cause the output to be $f\left(0^{\mathrm{n}_{1}}, y\right)$. While this is not necessarily the correct output (as $P_{1}$ 's input

\footnotetext{
${ }^{3}$ Note that in the semi-honest setting $P_{1}$ can in fact just send $b_{w} \oplus \lambda_{w}$ instead of sending his share; however, when we discuss malicious security we will find it necessary for $P_{1}$ to send his share and not $b_{w} \oplus \lambda_{w}$. Thus, we include this slight complication in the semi-honest setting to help ease our transition to malicious security.

${ }^{4}$ Note that the prior footnote about sending $b_{w} \oplus \lambda_{w}$ instead of the shares applies in this step as well.
} 
could be any $\left.x \leftarrow\{0,1\}^{\mathrm{n}_{1}}\right), \mathcal{S}_{2}$ can correct this by modifying the selection bits on the output gates of the circuit to contain $z=f(x, y)$ rather than $f\left(0^{\mathrm{n}_{1}}, y\right)$. The security of the encryption scheme guarantees that these modified selection bits do not provide the adversary with any distinguishing advantage.

More precisely, playing as $P_{1}$ and emulating the ideal functionalities, $\mathcal{S}_{2}$ proceeds as follows:

1. As $\mathcal{S}_{2}$ does not know $P_{1}$ 's input, it uses $0^{\mathrm{n}_{1}}$ instead.

2. $S_{2}$ sends $P_{2}^{*}$ 's input $y$ to the SFE ideal functionality and receives the function output $z=f(x, y)$ in return.

3. In performing the distributed garbling, for each of $P_{2}^{*}$ 's input wires, $\mathcal{S}_{2}$ receives the mask bits $\lambda_{w}$ (through the calls to $\mathcal{F}_{\mathbf{s s}}$ ), and for each gate $G_{\gamma}, \mathcal{S}_{2}$ receives the sub-keys $s_{\gamma, 0}^{2}$ and $s_{\gamma, 1}^{2}$ (through the calls to $\mathcal{F}_{\text {oshare }}$ ). $\mathcal{S}_{2}$ chooses all other mask bits uniformly at random except for the output masks (which $\mathcal{S}_{2}$ sets to 0 ).

Now, when the output wires are decrypted, $P_{2}^{*}$ expects to receive the correct output $z$. $\mathcal{S}_{2}$ accomplishes this as follows. Given inputs $x$ and $y, \mathcal{S}_{2}$ can compute, for each gate $G_{\gamma}$, the expected output $z_{\gamma}$ of that gate. Thus, for every gate $G_{\gamma}$ in the circuit, $\mathcal{S}_{2}$ modifies the construction of the garbled gate as follows: For each row $P[\gamma, j, k]$ of the garbled gate, $\mathcal{S}_{2}$ modifies the output of the $\mathcal{F}_{\text {gate }}$ calls to output $\left\langle z_{\gamma}\right\rangle=\left(\left\langle z_{\gamma}\right\rangle^{(1)},\left\langle z_{\gamma}\right\rangle^{(2)}\right)$ (instead of having $\mathcal{F}_{\text {gate }}$ output $\left.\left\langle\sigma_{\gamma, j, k}\right\rangle\right)$. This ensures that irrespective of which row is actually decrypted, the output of gate $G_{\gamma}$ will be $z_{\gamma}$ as in the ideal computation, and the sub-keys will be consistent with the selector bit.

This concludes the description of the simulator. We now argue that the simulated transcript is indistinguishable from the real one. The proof of simulation follows by a hybrid argument. We assume that all the gates have two inputs and a single output, and are topologically ordered, where the input gates come first, then the inner gates and finally the output gates.

For $i \in\{0, \ldots, \mathrm{q}\}$ we define the hybrid $H_{i}$ as follows: $H_{i}$ is the protocol execution in which the garbled gates indexed by $\mathrm{n}+1, \ldots, \mathrm{n}+i$ are constructed according to the real execution and the remaining $\mathrm{q}-i$ gates constructed according to the simulated execution. Note that $H_{0}$ corresponds to the real execution, and $H_{\mathrm{q}}$ to the simulated execution. So, it suffices to show that for $i \in\{0, \ldots, \mathrm{q}-1\}$, the neighboring hybrids $H_{i}$ and $H_{i+1}$ are indistinguishable.

Choose $i$ which maximizes the adversary's advantage, and let $G_{\gamma}$ refer to $G_{\mathrm{n}+i}$ (namely, $G_{\gamma}$ is the gate which is garbled as in the real execution in hybrid $H_{i}$ but replaced by a simulated gate in hybrid $\left.H_{i+1}\right)$. Denote by $\alpha$ and $\beta$ the input wires to $G_{\gamma}$ and by $\gamma$ the output wire. In $H_{i}$, the third component of the plaintext in the garbled row $P^{1}[\gamma, j, k]$ is $\left\langle\sigma_{\gamma, j, k}\right\rangle^{(1)}$, whereas in $H_{i+1}$ this component is $\left\langle z_{\gamma}\right\rangle^{(1)}$. The claim that this does not create a distinguishing advantage follows from the security of the encryption scheme. The argument is nearly identical to the argument presented by Lindell and Pinkas [LP09, pp. 183-187], and is thus not repeated.

This completes the proof.

\section{D.2 The Malicious Setting}

Intuitively, $\Pi_{\mathbf{2 P C}}\left(P_{1}, P_{2}\right)$, using the "authenticated" ideal functionalities from Section 3.3, is a maliciously secure protocol because the adversary is unable to cheat by modifying the authenticated shared bits. The only alternative is for the adversary to cheat by modifying his sub-keys, which would 
cause the protocol to abort. However, each garbled gate is permuted according to random shared bits, and therefore the adversary has no notion of which row will be decrypted on evaluation; if he corrupts $t$ rows in a given garbled gate, the probability of the protocol aborting is $t / 4$, independent of each party's input.

Theorem 4. Let $C$ be an arbitrary polynomial-size circuit. Then the protocol $\Pi_{\mathbf{2} \mathbf{P C}}\left(P_{1}, P_{2}\right)$, using authenticated hybrids as described above, securely evaluates the circuit $C$ in the presence of a (static) malicious adversary in the $\left(\mathcal{F}_{\text {const }}, \mathcal{F}_{\text {gate }}, \mathcal{F}_{\text {oshare }}, \mathcal{F}_{\text {rand }}, \mathcal{F}_{\text {ss }}\right)$-hybrid world.

Proof. We construct simulators $\mathcal{S}_{1}$ and $\mathcal{S}_{2}$ which simulate an adversary corrupting $P_{1}$ and $P_{2}$, respectively. In the following analysis, we ignore the negligible probability difference due to the direct attack on the authentication mechanism.

Simulator $\mathcal{S}_{1}$ : We simulate adversary $P_{1}^{*}$ corrupting party $P_{1}$ as follows. The simulator $\mathcal{S}_{1}$ chooses $0^{\text {n-n }}{ }^{-}$as $P_{2}$ 's input and then runs exactly as $P_{2}$ would. In addition, $\mathcal{S}_{1}$ extracts $P_{1}^{*}$ 's input $x$ through the calls to $\mathcal{F}_{\text {ss }}^{1}$ and passes $x$ to the ideal SFE functionality.

As $\mathcal{S}_{1}$ acts exactly as $P_{2}$ does (albeit on a different input), and $P_{1}^{*}$ receives no output, we need only show that the protocol aborts with equal probability across the two views.

Note that $P_{1}^{*}$ has three possible places in the protocol in which he can try to force the protocol to abort:

1. Sending an invalid sub-key in Step 5 or Step 6 of $\Pi_{\mathbf{2 P C}}$,

2. Inputting invalid or flipped sub-keys into the calls to $\mathcal{F}_{\text {oshare }}$, or

3. Encrypting the incorrect sub-key shares or using some arbitrary string as encryptions.

We claim that the probability of aborting due to any of the above attacks is independent of $P_{2}$ 's input. Clearly, if $P_{1}^{*}$ sends invalid sub-keys for his own input wires, the probability of aborting is independent of $P_{2}$ 's input. In the case that $P_{1}^{*}$ sends an invalid sub-key in Step 6 of $\Pi_{\mathbf{2 P C}}$, the probability of aborting is independent of $P_{2}$ 's input due to the masking by the (uniformly chosen) mask bit.

Now consider the case where $P_{1}^{*}$ corrupts $t$ rows in a given garbled gate. Note that even though $P_{1}^{*}$ can control which rows in the garbled gate table to corrupt, the probability that any given row is hit during evaluation is exactly $1 / 4$ (by the security of the point-and-permute method). Thus, the probability that a given bad row is hit is $t / 4$, independent of the bits on the incoming wires into the gate. Thus, as the probability of aborting is independent of $P_{2}$ 's input, the two views are perfectly indistinguishable.

Simulator $\mathcal{S}_{2}$ : We simulate adversary $P_{2}^{*}$ corrupting party $P_{2}$ as follows. The simulator $\mathcal{S}_{2}$ selects $0^{\mathrm{n}_{1}}$ as the input of $P_{1}$, and then proceeds to act as $P_{1}$ in $\Pi_{\mathbf{G C}}$. Then, $\mathcal{S}_{2}$ extracts $P_{2}^{*}$ 's input $y$ through the calls to $\mathcal{F}_{\mathrm{ss}}^{2}$ and passes $y$ to the ideal functionality, receiving back $f(x, y)$. Now, $\mathcal{S}_{2}$ continues executing as $P_{1}$, except it modifies its share for the output of $\mathcal{F}_{\text {gate }}$ on the output gates so that the selector bits for all rows in the output gates contain the appropriate bit from $f(x, y)$. In more detail, right before sending its share $G C^{1}$ at Step 4 of the protocol, $\mathcal{S}_{2}$ does the following:

- For each output gate $G_{\gamma}$, let $z_{\gamma}$ denote the correct output (i.e., the appropriate bit from $f(x, y)$ ) of the gate. Now, for each of the four rows of this gate, $\mathcal{S}_{2}$ modifies the original authenticated sharing $\left(\left\langle z_{\gamma, 1}\right\rangle^{(1)},\left\langle z_{\gamma, 2}\right\rangle^{(2)}\right)$ into a new sharing $\left(\left\langle z_{\gamma, 1}^{*}\right\rangle^{(1)},\left\langle z_{\gamma, 2}\right\rangle^{(2)}\right)$ that would be reconstructed to $z_{\gamma}$. Note that this is possible, since $\mathcal{S}_{2}$ has been emulating the $\mathcal{F}_{\text {gate }}$ functionality and has all the information necessary to construct new authenticated shares. In addition, $\mathcal{S}_{2}$ modifies the corresponding encryption in the garbled gate accordingly. 
$\mathcal{S}_{2}$ continues executing as $P_{1}$, and outputs whatever $P_{2}^{*}$ outputs.

We now prove that the view of the adversary when communicating with $\mathcal{S}_{2}$ versus the view when communicating with a real $P_{1}$ is computationally indistinguishable. We show this by constructing a set of hybrids and proving indistinguishability between them.

- $H_{0}$ : The same as the real execution with $P_{1}$.

- $H_{1}$ : The same as $H_{0}$, except the output of $\mathcal{F}_{\text {gate }}$ in each output gate is modified to be equal to an authenticated sharing of the correct output from $f(x, y)$.

- $H_{2}$ : The same as $H_{1}$, except input $0^{\mathrm{n}_{1}}$ is used instead of $P_{1}$ 's real input.

We first show that hybrids $H_{0}$ and $H_{1}$ are computationally indistinguishable. This follows from the security of the underlying garbling scheme; the only difference here is that $P_{2}^{*}$ can try to force the protocol to abort. However, by the security of the authenticated bit sharing scheme, the output of $\mathcal{F}_{\text {gate }}$ towards $P_{2}^{*}$ provides no information about the underlying selector bit's value, and thus $P_{2}^{*}$ acts independently of the value $\sigma_{\gamma, i, j}$.

The indistinguishability between $H_{1}$ and $H_{2}$ can be shown similarly to the case in which $P_{1}$ is corrupted.

Note that $H_{0}$ is simply the real execution of the protocol, and $H_{2}$ is the execution of the simulator $\mathcal{S}_{2}$. Thus, as each hybrid is (at least) computationally indistinguishable from its neighboring hybrids, we conclude that the protocol is secure.

\section{E Proof of Theorem 1}

Theorem. Let $C$ be an arbitrary polynomial-size circuit and let $\mathbb{G}$ be a cyclic group with prime order. Given access to ideal functionalities $\mathcal{F}_{\text {const }}, \mathcal{F}_{\text {gate }}, \mathcal{F}_{\text {oshare }}, \mathcal{F}_{\text {ot }}, \mathcal{F}_{\text {rand }}$, and $\mathcal{F}_{\text {ss }}$, and assuming that the decisional Diffie-Hellman problem is hard in $\mathbb{G}$, then $\Pi_{3 \mathbf{P C}}^{m}\left(P_{1}, P_{2}, P_{3}\right)$ securely computes the circuit $C$ in the presence of an adversary corrupting an arbitrary number of parties.

Proof (Sketch). The proof is similar to prior work in two-party garbling schemes based on cut-andchoose [LP07, LP11, sS11].

We make use of the following lemma:

Lemma. Consider garbled gate $G_{\gamma}$ with input wires $\alpha$ and $\beta$, and let $X_{w, b}=\left(s_{w, b}^{1}, s_{w, b}^{2}, b \oplus \lambda_{w}\right)$, for $w \in\{\alpha, \beta\}$ denote the valid keys. Fix a (valid) key $X_{w, b}$ for some fixed $w$ and $b$. Let $\bar{X}_{w, b}$ be equal to $X_{w, b}$ except that two of the three components (i.e., the sub-keys and selector bit) are altered arbitrarily. Then using key $\bar{X}_{w, b}$ to decrypt $G_{\gamma}$ causes a decryption failure with all but negligible probability.

Proof. This follows directly from our encryption scheme and garbling scheme.

What this lemma says is that for a given garbled gate, a sub-key / selector bit combo can only be used correctly on a single row of the garbled table, and modifying some (but not all) of the components results in a decryption failure; thus an adversary must change both the sub-key and permutation bit accordingly for the garbled gate to successfully decrypt. Note that in the two-party secure computation protocol described in Section 3 we enforce the above by authenticating all of the selector bits (thus preventing any malicious party from altering these). However, the authenticated sharing protocol only works between two parties, namely the parties doing the distributed garbling. 
Thus, we need a way for the evaluator to gain confidence in the sub-key / selector bit combos sent to him by $P_{1}$ and $P_{2}$. We do this by utilizing the Diffie-Hellman pseudorandom synthesizer trick of Lindell and Pinkas [LP11]. This enforces that $P_{1}$ and $P_{2}$ are consistent in the sub-keys they send to $P_{3}$, and because at least one sub-key is correct, the adversary can at most cause $P_{3}$ to abort.

There are six possible (interesting) corruption cases. However, due to symmetries, we only need to consider four of them.

The adversary corrupts parties $P_{1}$ and $P_{2}$. We need to construct a simulator $\mathcal{S}$ with access to the adversary $\mathcal{A}$ (who controls $P_{1}$ and $P_{2}$ ) and a trusted third party which computes $f(x, y, z$ ) given inputs $x, y$, and $z$. The simulator $\mathcal{S}$ is constructed as follows: $\mathcal{S}$ invokes the adversary $\mathcal{A}$, and the runs as $P_{3}$ would until Step 10. Here, $\mathcal{S}$ uses the witnesses $a_{w, b_{w}}^{i}$ send by $P_{1}$ and $P_{2}$ to $\mathcal{F}_{\text {zkpok }}$ to extract their inputs. $\mathcal{S}$ then feeds these inputs to the trusted third party, receiving back $f(x, y, z)$. $\mathcal{S}$ continues to run as $P_{3}$ would, and halts, outputting whatever $\mathcal{A}$ outputs.

We now argue that the adversary's view in the real and ideal worlds are computationally indistinguishable. The proof closely follows existing work [LP11, pp. 17-21], and thus we only give some intuition here.

Note that if $\mathcal{A}$ tries to cheat in Step 5, he will get caught in the cut-and-choose step with high probability. Similarly, if $\mathcal{A}$ tries to send different keys in Step 8 (i.e., the "input inconsistency" attack), he will get caught in Step 10 when proving the consistency of the sub-keys sent.

The adversary corrupts parties $P_{1}$ and $P_{3}$. We again demonstrate a simulator, this time with $\mathcal{A}$ controlling parties $P_{1}$ and $P_{3}$. This is similar to the two-party case where $P_{2}$ is corrupted. The main challenge is that the simulator needs to construct "fake" garbled circuits in order for $\mathcal{A}$ to output the correct output; however, as shown in the proof of our two-party protocol, such a simulator exists. Thus, the simulator $\mathcal{S}$ is constructed as follows: $\mathcal{S}$ invokes the adversary $\mathcal{A}$, and runs as $P_{2}$ would up until Step 6. $\mathcal{S}$ can extract both $P_{1}$ 's input $x$ and its mask bits $\lambda_{w, j}$ through $P_{1}$ 's calls to $\mathcal{F}_{\text {ss }}^{1}$ in Step 4 and Step 2, Likewise, in Step 5, $\mathcal{S}$ extracts $P_{3}$ 's input $z$ through the calls to $\mathcal{F}_{\text {ot }} \cdot \mathcal{S}$ then passes $x$ and $z$ to the trusted third party, learning $f(x, y, z)$. In Step $6, \mathcal{S}$ chooses $\rho \leftarrow \$\{0,1\}^{s}$. Then for $j \in\{1, \ldots, s\}, \mathcal{S}$ proceeds as follows: If $\rho_{j}=0, \mathcal{S}$ uses the simulator that is known to exist for the two-party circuit garbling protocol to construct garbled circuits which output $f(x, y, z)$. Otherwise, $\mathcal{S}$ acts as $P_{2}$ would. $\mathcal{S}$ continues to act as $P_{2}$ would, except that in Step 7 it sets the output of $\mathcal{F}_{\text {cf }}$ to be equal to the $\rho$ chosen above. Finally, $\mathcal{S}$ halts, outputting whatever $\mathcal{A}$ outputs.

The main intuition here is that, since $P_{1}$ learns nothing about the portion of the circuit garbled by $P_{2}$, this reduces to the two-party setting where $P_{2}$ is corrupt. Recall that by the security of our garbling protocol, $P_{1}$ can only construct circuits that cause the evaluator to abort. If $P_{1}$ tries to cheat in Step 5 by exchanging invalid mask shares, $P_{2}$ will detect this with high probability, and likewise for Step 8 .

The adversary corrupts parties $P_{2}$ and $P_{3}$. The analysis here is very similar to the case where parties $P_{1}$ and $P_{3}$ are corrupt.

The adversary corrupts party $P_{1}$. We construct a simulator $\mathcal{S}$ with access to an adversary $\mathcal{A}$ controlling $P_{1}$ as follows: $\mathcal{S}$ invokes the adversary $\mathcal{A}$, and runs as $P_{2}$ and $P_{3}$ would, extracting $P_{1}$ 's input $x$ through the calls to $\mathcal{F}_{\text {ss }}^{1}$ in Step $4 . \mathcal{S}$ passes $x$ to the trusted third party, learning $f(x, y, z)$, and halts, outputting whatever $\mathcal{A}$ outputs.

As $\mathcal{S}$ acts exactly as $P_{2}$ and $P_{3}$ would, and $\mathcal{A}$ gets no output, we need only show that the probability that $\mathcal{A}$ aborts in both the real and ideal world is identical. In fact, this follows from our maliciously secure two-party protocol and the security of the input-consistency checks. 
The adversary corrupts party $P_{2}$. The analysis here is very similar to the case where party $P_{1}$ is corrupt.

The adversary corrupts party $P_{3}$. We construct a simulator $\mathcal{S}$ with access to an adversary $\mathcal{A}$ controlling $P_{3}$ as follows: $\mathcal{S}$ invokes the adversary $\mathcal{A}$, and runs as $P_{1}$ and $P_{2}$ would, extracting $P_{3}$ 's input $z$ through the $\mathcal{F}_{\text {ot }}$ calls in Step 5, $\mathcal{S}$ then hands $z$ to the trusted third party, who returns $f(x, y, z)$. In Step 6, $\mathcal{S}$ chooses $\rho \leftarrow \$\{0,1\}^{s}$, and then for $j \in\{1, \ldots, s\} \mathcal{S}$ proceeds as follows: If $\rho_{j}=0, \mathcal{S}$ constructs a distributed garbled circuit which outputs $f(x, y, z)$, otherwise $\mathcal{S}$ proceeds as normal. Then, in Step 7, $\mathcal{S}$ fixes the output of $\mathcal{F}_{\text {cf }}$ to be $\rho$. For the rest of the protocol, $\mathcal{S}$ acts as $P_{1}$ and $P_{2}$ would, and eventually halts, outputting what $\mathcal{A}$ outputs.

The analysis is very similar to prior work [LP11, pp. 22-23].

\section{F Proof of Theorem 2}

Theorem. Let $C$ be an arbitrary polynomial-size circuit and let $\mathbb{G}$ be a cyclic group with prime order. Given access to ideal functionalities $\mathcal{F}_{\text {const }}, \mathcal{F}_{\text {gate }}, \mathcal{F}_{\text {oshare }}, \mathcal{F}_{\text {ot }}, \mathcal{F}_{\text {rand }}$, and $\mathcal{F}_{\text {ss }}$, and assuming that the decisional Diffie-Hellman problem is hard in $\mathbb{G}$, then $\Pi_{\mathbf{3} \mathbf{P C}}^{\text {-lindell }}\left(P_{1}, P_{2}, P_{3}\right)$ securely computes the circuit $C$ in the presence of an adversary corrupting an arbitrary number of parties.

Proof (Sketch). The analysis here is nearly identical to the proof in Appendix E as well as the proof for the two-party case [Lin13], and thus we just present the simulators for each corruption case.

The adversary corrupts parties $P_{1}$ and $P_{2}$. The simulator $\mathcal{S}$ is constructed as follows: $\mathcal{S}$ invokes the adversary $\mathcal{A}$, and the runs as $P_{3}$ would, using input $0^{\mathrm{n}-\mathrm{n}_{2}}$, until Step 11. Here, $\mathcal{S}$ uses the witnesses $a_{w, b_{w}}^{i}$ send by $P_{1}$ and $P_{2}$ for the zero-knowledge proof-of-knowledge to extract their inputs. $\mathcal{S}$ then feeds these inputs to the trusted third party, receiving back $f(x, y, z)$. $\mathcal{S}$ continues to run as $P_{3}$ would, and halts, outputting whatever $\mathcal{A}$ outputs.

The adversary corrupts parties $P_{1}$ and $P_{3}$. The simulator $\mathcal{S}$ is constructed as follows: $\mathcal{S}$ invokes the adversary $\mathcal{A}$, and runs as $P_{2}$ would up until Step 6. $\mathcal{S}$ can extract both $P_{1}$ 's input $x$ and its mask bits $\lambda_{w, j}$ through $P_{1}$ 's calls to $\mathcal{F}_{\text {ss }}^{1}$ in Step 2 and Step 4 . Likewise, in Step 5, $\mathcal{S}$ extracts $P_{3}$ 's input $z$ through the calls to $\mathcal{F}_{\text {ot }}$. $\mathcal{S}$ then passes $x$ and $z$ to the trusted third party, learning $f(x, y, z)$. In Step 6, $\mathcal{S}$ chooses $\rho \leftarrow \$\{0,1\}^{s}$. Then for $j \in\{1, \ldots, s\}, \mathcal{S}$ proceeds as follows: If $\rho_{j}=0, \mathcal{S}$ uses the simulator that is known to exist for the two-party circuit garbling protocol to construct garbled circuits which output $f(x, y, z)$. Otherwise, $\mathcal{S}$ acts as $P_{2}$ would. $\mathcal{S}$ continues to act as $P_{2}$ would, except that in Step 7 it sets the output of $\mathcal{F}_{\mathbf{c f}}$ to be equal to the $\rho$ chosen above. Finally, $\mathcal{S}$ halts, outputting whatever $\mathcal{A}$ outputs.

The adversary corrupts parties $P_{2}$ and $P_{3}$. The analysis here is exactly the same as the case where parties $P_{1}$ and $P_{3}$ are corrupt.

The adversary corrupts party $P_{1}$. We construct a simulator $\mathcal{S}$ with access to an adversary $\mathcal{A}$ controlling $P_{1}$ as follows: $\mathcal{S}$ invokes the adversary $\mathcal{A}$, and runs as $P_{2}$ and $P_{3}$ would, extracting $P_{1}$ 's input $x$ through the calls to $\mathcal{F}_{\text {ss }}^{1}$ in Step $4 . \mathcal{S}$ passes $x$ to the trusted third party, learning $f(x, y, z)$. For the rest of the protocol, $\mathcal{S}$ acts as $P_{2}$ and $P_{3}$ would, and eventually halts, outputting whatever $\mathcal{A}$ outputs.

The adversary corrupts party $P_{2}$. The analysis here is exactly the same as the case where party $P_{1}$ is corrupt. 


\begin{tabular}{cccc}
\hline Step & Exponentiations & Hybrids Calls & Symmetric Ops \\
\hline 1 & & & \\
2 & $4 \ell+2(3 s)$ & $\left(2 \ell \cdot \mathcal{F}_{\mathbf{s s}}+\left(\ell^{\prime}+\mathbf{q}\right) \cdot \mathcal{F}_{\text {rand }}+4 \ell \cdot H\right) \cdot(3 s)$ & \\
3 & & $\left(4 \mathbf{q} \cdot \mathcal{F}_{\text {gate }}+8 \mathbf{q} \cdot \mathcal{F}_{\text {oshare }}\right) \cdot(3 s)$ & $8(3 s) \mathbf{q}$ \\
4 & $2 \ell \cdot \mathcal{F}_{\mathbf{s s}}$ & \\
5 & & $\left(2 \ell^{\prime} \cdot \mathcal{F}_{\mathbf{~ o t}}\right) \cdot(3 s)$ & \\
6 & & $\mathcal{F}_{\mathbf{c f}}$ & \\
7 & $3 s$ & & $4(3 s) \mathbf{q}$ \\
8 & & $\left(2 \ell \cdot H+2 \ell \cdot \mathcal{F}_{\mathbf{z k p o k}}\right) \cdot(3 s)$ & $(3 s) \mathbf{q}$ \\
9 & & & \\
10 & &
\end{tabular}

Table 1: Computational cost for each step of $\Pi_{3 \mathbf{P C}}^{m}\left(P_{1}, P_{2}, P_{3}\right)$.

The adversary corrupts party $P_{3}$. We construct a simulator $\mathcal{S}$ with access to an adversary $\mathcal{A}$ controlling $P_{3}$ as follows: $\mathcal{S}$ invokes the adversary $\mathcal{A}$, and runs as $P_{1}$ and $P_{2}$ would, extracting $P_{3}$ 's input $z$ through the $\mathcal{F}_{\text {ot }}$ calls in Step 5, $\mathcal{S}$ then hands $z$ to the trusted third party, who returns $f(x, y, z)$. In Step $6, \mathcal{S}$ chooses $\rho \leftarrow \$\{0,1\}^{s}$, and then for $j \in\{1, \ldots, s\} \mathcal{S}$ proceeds as follows: If $\rho_{j}=0, \mathcal{S}$ constructs a distributed garbled circuit which outputs $f(x, y, z)$, otherwise $\mathcal{S}$ proceeds as normal. Then, in Step 7, $\mathcal{S}$ fixes the output of $\mathcal{F}_{\text {cf }}$ to be $\rho$. For the rest of the protocol, $\mathcal{S}$ acts as $P_{1}$ and $P_{2}$ would, and eventually halts, outputting whatever $\mathcal{A}$ outputs.

\section{G Concrete Efficiency of the 3PC Protocol}

For simplicity we assume each party's input has length $\ell$. Since we apply the XOR-tree technique to $P_{3}$ 's input, we let $\ell^{\prime}=\max \{4 \ell, 8 s\}$ be the new input length. Finally, we redefine $s$ in this section to be the statistical security parameter; namely, the adversary can succeed in cheating with probability at most $2^{-s}$.

Protocol Based on Cut-and-Choose. Table 1 details the specific computational cost of each step for $\Pi_{3 \mathbf{P C}}^{m}\left(P_{1}, P_{2}, P_{3}\right)$. Note that these numbers are across all parties; the actual per-party cost is less. Each of the hybrid calls can be instantiated efficiently using known techniques: $\mathcal{F}_{\text {cf }}$ can be instantiated very efficiently in the random oracle model requiring only three oracle calls, $\mathcal{F}_{\text {zkpok }}$ can be instantiated using $3 s / 2+18$ exponentiations [LP11, pg. 36], and $\mathcal{F}_{\text {ot }}$ can be computed using three exponentiations PVW08].

Protocol Based on Lindell's Protocol. The concrete computational cost for this protocol are similar to the ones above, except $s$ in this case is smaller to achieve the same level of security. However, we must run the above protocol as a sub-protocol. See Table 2 for the concrete efficiency counts.

Comparison with SPDZ. We compare our three-party protocol with the SPDZ protocol BBDOZ11, DPSZ12, $\mathrm{DKL}^{+} 12, \mathrm{DKL}^{+} 13$, KSS13, an efficient MPC protocol which works for $n$ parties and arbitrary corruptions over arithmetic circuits, and follows the preprocessing paradigm. SPDZ represents the state-of-the-art at the time of writing in terms of efficiency in the multi-party setting. Here we focus on the differences between both SPDZ and our protocol, and discuss their strengths and weaknesses. Due to the different characteristics of each protocol (e.g., arithmetic versus boolean, 


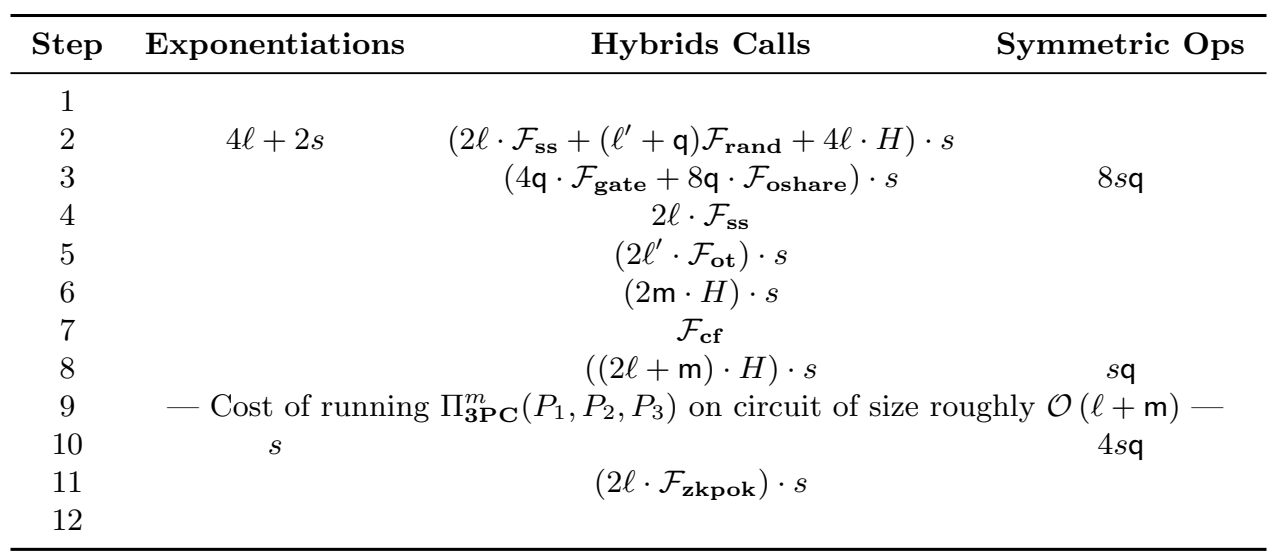

Table 2: Computational cost for each step of $\Pi_{\mathbf{3} \mathbf{P C}}^{m-\text { lindell }}\left(P_{1}, P_{2}, P_{3}\right)$.

linear versus constant round, etc.), these protocols are somewhat "incomparable". However, we hope to give a general idea of the efficiency trade-offs of both protocols.

There are several key differences between the SPDZ protocol and our own. For one, SPDZ works over arithmetic circuits, whereas our protocol works over boolean circuits.5 In terms of communication, the SPDZ protocol requires rounds linear in the depth of the circuit, whereas our protocol is constant-round. While it is difficult to compare the impact of this without an implementation and experiments, it seems intuitive that as the latency between machines increases, the cost of each additional communication round increases as well; this intuition has been backed up by experiments in the semi-honest setting [SZ13.

Finally, we consider the start-to-finish execution time (i.e., including the cost of preprocessing) for running an AES circuit. The preprocessing in our protocol is basically that found in the TinyOT protocol [NNOB12], and, using the numbers presented there, is fairly efficient (around 1 minute [NNOB12, Figure 21]). Efficiency comes from the fact that the preprocessing is only between two parties, namely, the circuit generators. The on-line running time is conjectured to be around that of maliciously secure two-party protocols using cut-and-choose.

The SPDZ protocol, on the other hand, has a very efficient (information-theoretic) online phase but a much costlier offline phase (around 17 minutes for three parties [DKL ${ }^{+} 12$, Table 2]). In addition, it has a one-time setup phase which is very costly: the parties need to execute an MPC protocol for a circuit which generates a key pair with the secret key secret-shared among the parties. Executing this on its own would likely eclipse the running time of our protocol 6 Thus, given preprocessing, it seems likely that SPDZ would out-perform our protocol; however, in the setting of executing the protocol from start to finish, we conjecture that our protocol would be more efficient.

Finally, our protocol is most efficient in the random oracle model, whereas SPDZ works in the standard model.

\footnotetext{
${ }^{5}$ Damgård and Zakarias [DZ13] develop a SPDZ-like protocol for Boolean circuits; however, its practical efficiency is unclear.

${ }^{6}$ We note that the work of Damgård et al. $\mathrm{DKL}^{+} 13$. presents an efficient protocol for this one-time setup phase in the weaker covert security model [AL07.
} 


\section{Changelog}

- Version 1.1 (June 27, 2014)

- Corrected computation/communication costs.

- Cleaned up some writing.

- Updated motivation for 3PC.

- Version 1.0 (February 19, 2014)

- First release. 